\title{
Disruption of STAT3 signalling promotes KRAS-induced lung tumorigenesis
}

\author{
Beatrice Grabner ${ }^{1}$, Daniel Schramek ${ }^{2}{ }^{\dagger}$, Kristina M. Mueller ${ }^{1,3}$, Herwig P. Moll ${ }^{4}$, Jasmin Svinka ${ }^{5}$, \\ Thomas Hoffmann ${ }^{6}$, Eva Bauer ${ }^{1}$, Leander Blaas ${ }^{7}$, Natascha Hruschka', Katalin Zboray ${ }^{1}$, Patricia Stiedl ${ }^{1}$, \\ Harini Nivarthi ${ }^{1} \uparrow$, Edith Bogner ${ }^{1}$, Wolfgang Gruber ${ }^{8}$, Thomas Mohr ${ }^{5}$, Ralf Harun Zwick ${ }^{9}$, Lukas Kenner ${ }^{1,10,11}$, \\ Valeria Poli ${ }^{12}$, Fritz Aberger ${ }^{8}$, Dagmar Stoiber ${ }^{1,4}$, Gerda Egger ${ }^{10}$, Harald Esterbauer ${ }^{13}$, Johannes Zuber ${ }^{6}$, \\ Richard Morigg ${ }^{1,3}$, Robert Eferl ${ }^{5}$, Balázs Györffy ${ }^{14,15,16}$, Josef M. Penninger ${ }^{2}$, Helmut Popper ${ }^{17}$ \\ \& Emilio Casanova ${ }^{1,18}$
}

STAT3 is considered to play an oncogenic role in several malignancies including lung cancer; consequently, targeting STAT3 is currently proposed as therapeutic intervention. Here we demonstrate that STAT3 plays an unexpected tumour-suppressive role in KRAS mutant lung adenocarcinoma ( $A C$ ). Indeed, lung tissue-specific inactivation of Stat3 in mice results in increased Kras ${ }^{\mathrm{G} 12 \mathrm{D}}$-driven $\mathrm{AC}$ initiation and malignant progression leading to markedly reduced survival. Knockdown of STAT3 in xenografted human AC cells increases tumour growth. Clinically, low STAT3 expression levels correlate with poor survival and advanced malignancy in human lung $A C$ patients with smoking history, which are prone to KRAS mutations. Consistently, KRAS mutant lung tumours exhibit reduced STAT3 levels. Mechanistically, we demonstrate that STAT3 controls NF-KB-induced IL-8 expression by sequestering NF- $\mathrm{KB}$ within the cytoplasm, thereby inhibiting IL-8-mediated myeloid tumour infiltration and tumour vascularization and hence tumour progression. These results elucidate a novel STAT3-NF-KB-IL-8 axis in KRAS mutant AC with therapeutic and prognostic relevance.

\footnotetext{
${ }^{1}$ Ludwig Boltzmann Institute for Cancer Research (LBI-CR), Vienna 1090, Austria. ${ }^{2}$ Institute of Molecular Biotechnology of the Austrian Academy of Sciences (IMBA), Vienna 1030, Austria. ${ }^{3}$ Institute of Animal Breeding and Genetics, University of Veterinary Medicine, Vienna 1210 and Medical University Vienna, Vienna 1090, Austria. ${ }^{4}$ Institute of Pharmacology, Medical University of Vienna, Vienna 1090, Austria. ${ }^{5}$ Institute of Cancer Research \& Department of Internal Medicine I, Comprehensive Cancer Center, Medical University of Vienna, Vienna 1090, Austria. ${ }^{6}$ Research Institute of Molecular Pathology (IMP), Dr. Bohr Gasse 7, Vienna 1030, Austria. ${ }^{7}$ Department of Biosciences and Nutrition, Center for Innovative Medicine, Karolinska Institutet, Novum, Huddinge 141 83, Sweden. ${ }^{8}$ Department of Molecular Biology, Paris-Lodron University of Salzburg, Salzburg 5020, Austria. ${ }^{9}$ Department of Internal Medicine, Karl Landsteiner University, 3430 Tulln, Austria. ${ }^{10}$ Clinical Institute of Pathology, Medical University of Vienna, Vienna 1090, Austria. ${ }^{11}$ Unit of Pathology of Laboratory Animals (UPLA), University of Veterinary Medicine Vienna, 1210 Vienna, Austria. ${ }^{12}$ Molecular Biotechnology Center (MBC), Department of Genetics, Biology and Biochemistry, University of Turin, Turin 10126, Italy. ${ }^{13}$ Department of Laboratory Medicine, Medical University of Vienna, Vienna 1090 , Austria. ${ }^{14}$ MTA TTK Lendület Cancer Biomarker Research Group, Budapest 1117, Hungary. ${ }^{15}$ 2nd Department of Pediatrics, Semmelweis University, Budapest 1094, Hungary. ${ }^{16}$ MTA-SE Pediatrics and Nephrology Research Group, Budapest 1083, Hungary. ${ }^{17}$ Institute of Pathology, Research Unit Molecular Lung and Pleura Pathology, Medical University of Graz, Graz 8036, Austria. ${ }^{18}$ Department of Physiology, Center of Physiology and Pharmacology, Comprehensive Cancer Center, Medical University of Vienna, Vienna 1090, Austria. † Present addresses: Howard Hughes Medical Institute, Laboratory of Mammalian Cell Biology and Development, Rockefeller University, 10065 New York, USA (D.S.); CeMM Research Center for Molecular Medicine of the Austrian Academy of Sciences, 1090 Vienna, Austria (H.N.). Correspondence and requests for materials should be addressed to E.C. (email: emilio.casanova@lbicr.lbg.ac.at).
} 
ung cancer is still the leading cause of cancer deaths worldwide $^{1}$. The most frequent genetic alterations found in lung adenocarcinomas (ACs) are missense mutations and amplifications of kirsten rat sarcoma viral oncogene (KRAS) and epidermal growth factor receptor (EGFR) in about 30\% and $20 \%$ of all Caucasian cases, respectively ${ }^{2,3}$. Although several targeted anti-EGFR therapies are effective in patients with EGFR mutations, oncogenic KRAS is still considered as an undruggable target. To elucidate further treatment strategies, the focus has therefore shifted towards KRAS-cooperating and downstream signalling pathways. Signal transducer and activator of transcription 3 (STAT3) represents one important transcription factor thought to cooperate with rat sarcoma viral oncogene (RAS) mutations during tumorigenesis ${ }^{4,5}$. STAT3 is activated in response to several cytokines and growth factors, such as interleukin-6 (IL-6), oncostatin-M (OSM) and epidermal growth factor (EGF). Upon binding of the ligand to its cognate receptor, STAT3 becomes tyrosine-phosphorylated and forms homodimers or heterodimers before translocating into the nucleus to induce the transcription of several target genes implicated in cell cycle regulation, apoptosis, angiogenesis, tumour invasion and metastasis 6 . Depending on the oncogenic driver mutation or on the cell type, STAT3 has been reported to play either pro-oncogenic ${ }^{4,5,7}$ or tumour-suppressive roles in vivo and in vitro ${ }^{8-12}$. Interestingly, STAT3 was shown to be activated in $54 \%$ of lung AC patient samples and human lung cancer cell lines ${ }^{13-15}$. Furthermore, based on in vitro and xenograft models, STAT3 is thought to play a tumour-promoting role in non-smallcell lung cancer (NSCLC) and during acquired drug resistance ${ }^{13,14,16-18}$

Here, we investigated the role of STAT3 during oncogenic Kras-driven lung tumorigenesis using the Cre-inducible LoxStop-Lox-Kras ${ }^{G 12 D /+}$ knock-in lung cancer mouse model ${ }^{19}$ and a human xenograft model. Surprisingly, our results show that STAT3 functions as tumour suppressor in Kras ${ }^{\mathrm{G} 12 \mathrm{D} /+}$ lung tumours as well as KRAS mutant human AC cell lines. Deletion of STAT3 in Kras ${ }^{\mathrm{G} 12 \mathrm{D} /}+$ lung tumours and human AC resulted in an increased tumour growth, higher tumour grade, increased vascularization, changes in the tumour microenvironment and significantly reduced survival. Mechanistically, we show that genetic ablation of Stat3 in murine as well as knockdown of STAT3 in human cells resulted in an increase of nuclear factorkappa B $(N F-\kappa B)$-induced expression of the pro-angiogenic chemokine ligand 1 (CXCL1; murine orthologue to the human $I L-8)$, thereby promoting tumour growth. Pharmacological inhibition of CXCL1's cognate receptor CXCR2 normalizes tumour vascularization and microenvironment and reduces tumour burden. Thus, STAT3 functions as tumour suppressor by sequestering $\mathrm{NF}-\kappa \mathrm{B}$ in the cytoplasm and thereby reducing $\mathrm{NF}-\kappa \mathrm{B}$-dependent CXCL1 transcription.

\section{Results}

STAT3 suppresses Kras-induced lung tumorigenesis. First, we investigated the activation status of STAT3 within murine Kras ${ }^{G 12 D}$-driven lung tumours. In all, $20-30 \%$ of tumour cells showed active STAT3 (tyrosine 705 phosphorylated STAT3) throughout tumour development. ELISA analysis revealed increased expression of STAT3-activating cytokines such as EGF, OSM and IL-6 in lungs harbouring Kras ${ }^{G 12 D}$-driven tumours compared with healthy control lungs (Supplementary Fig. 1a,b).

To functionally test the role of STAT3 during Kras ${ }^{G 12 D}$-driven lung tumour formation, we crossed the Kras ${ }^{L L L}-G 12 D /+\operatorname{strain}^{19}$ with conditional Stat $3^{\mathrm{fl} / \mathrm{fl}}$ mice ${ }^{20}$. Lung epithelial-specific activation of oncogenic Kras ${ }^{\mathrm{G} 12 \mathrm{D}}$ and concomitant deletion of Stat3 was achieved by adenoviral delivery of Cre-recombinase (AdCre) through inhalation of 8 - to 10 -week-old Stat $3^{\text {fl/fl. }}:$ ras $^{L S L-G 12 D /+}$ mice (Stat3 ${ }^{\text {Lep } / \Delta L e p}: K r a s^{G 12 D /}+$ hereafter; 4 Lep: deleted in the lung epithelium). Efficient deletion of Stat3 in lung tumour cells was confirmed by immunohistochemistry (IHC) of total STAT3 (Supplementary Fig. 1c). Notably, genetic ablation of Stat3 resulted in a markedly shortened survival. Although Stat ${ }^{\text {LLep/ }}$ ${ }^{\triangle L e P}: \mathrm{Kras}^{\mathrm{G} 12 \mathrm{D} /+}$ males showed a median survival of 118 days post AdCre infection, the control Stat $3^{+1+}: \mathrm{Kras}^{\mathrm{G12D} /+}$ male littermates survived 175 days (Fig. 1a). Interestingly, heterozygous loss of STAT3 within the tumours (Stat3 $\left.{ }^{\text {Lep } /+}: \operatorname{Kras}^{G 12 D /+}\right)$ led to the

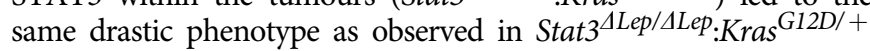
animals with a median survival of 108 days. Of note, female

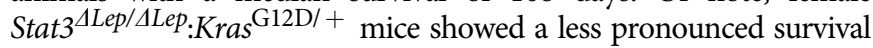
disadvantage when compared with their female littermate controls (Supplementary Fig. 1d).

We performed tumour analysis in male mice at three different stages: 6,10 and 13 weeks post AdCre-induction. Stat $3^{\text {Lep/ALep. }}$ $\mathrm{Kras}^{\mathrm{G} 12 \mathrm{D} /+}$ mice revealed a dramatically increased tumour burden compared with littermate controls at all time points (Fig. 1b). Already 6 weeks after tumour induction, Stat $3^{\text {LLep/ALep: }}$ Kras ${ }^{G 12 D /+}$ mice showed an increased number of hyperplasia (including multifocal pneumocyte hyperplasia and adenoma) as well as malignant in situ ACs compared with control Kras ${ }^{G 12 D /+}$ animals (Fig. 1c and Supplementary Fig. 1e). At 13 weeks post AdCre-induction, the size of ACs spreading throughout the whole lung precluded analysis of individual tumours. Therefore, we assessed tumour area/lung area and observed significantly increased areas of multifocal hyperplasia and in situ ACs in Stat ${ }^{\text {LLep/LLep }}: \mathrm{Kras}^{\mathrm{G} 12 \mathrm{D} /+}$ mice compared with control animals (Supplementary Fig. 1e). Of note, small areas of invasive ACs were observed in both experimental groups at 13 weeks post AdCre inhalation (Supplementary Fig. 1e). In line with these observations, Stat3-deficient multifocal hyperplasia at 6 and 13 weeks as well as in situ ACs at 6 weeks post AdCre infection displayed significantly enhanced cell proliferation compared with tumours from Stat3-proficient control littermates (Supplementary Fig. 1f), whereas the low amount of apoptosis observed in the Kras ${ }^{G 12 D}$-driven lung tumours was not altered upon genetic ablation of STAT3 (refs 21,22; Supplementary Fig. 1f). In addition, xenograft experiments using the human AC cell line A549, which carries a KRAS mutation in the same amino acid as our mouse lung cancer model (G12S), showed increased tumour growth upon STAT3 knockdown, thus confirming the observations made in the mouse model (Fig. 1d and Supplementary Fig. 2a). Together, these data show that STAT3 suppresses

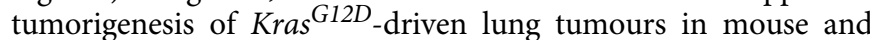
human xenograft models.

Given these results, we hypothesized that human AC with a molecular signature similar to Stat3-deficient tumours might have a worse prognosis. To test this idea, microarray data were used to identify differentially expressed genes between Stat3-proficient and Stat3-deficient tumours isolated 13 weeks post induction. Subsequent gene set enrichment analysis (GSEA) revealed that Stat3 ${ }^{\text {Lep/ALep }: K r a s}{ }^{G 12 D /+}$ tumours indeed showed significant positive enrichment for genes associated with poor prognosis in human AC lung cancers and, conversely, significant negative enrichment for genes associated with good prognosis in humans (Supplementary Fig. 1g; ref. 23).

These data show that Stat3-deficient tumours share a transcriptional profile with advanced progression and poor prognosis observed in human AC. In addition, the gene expression profile of murine Kras $^{G 12 D}$ tumours devoid of STAT3 signalling significantly overlapped with human KRAS mutant lung $\mathrm{AC}$, and, conversely, murine Kras ${ }^{\mathrm{G} 12 D}$ tumours competent of STAT3 signalling showed a significant enrichment for genes usually downregulated in human KRAS mutant lung AC (Supplementary Fig. 1h; ref. 24). This result led us to test 
a

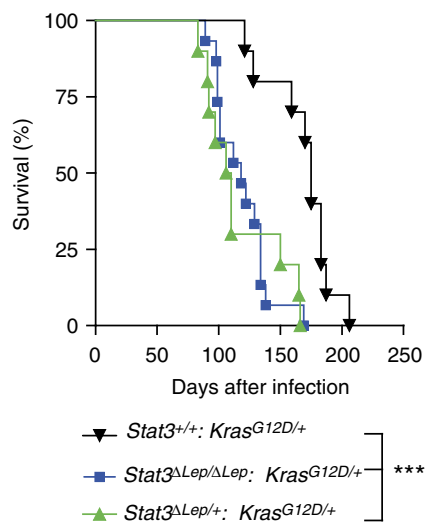

b

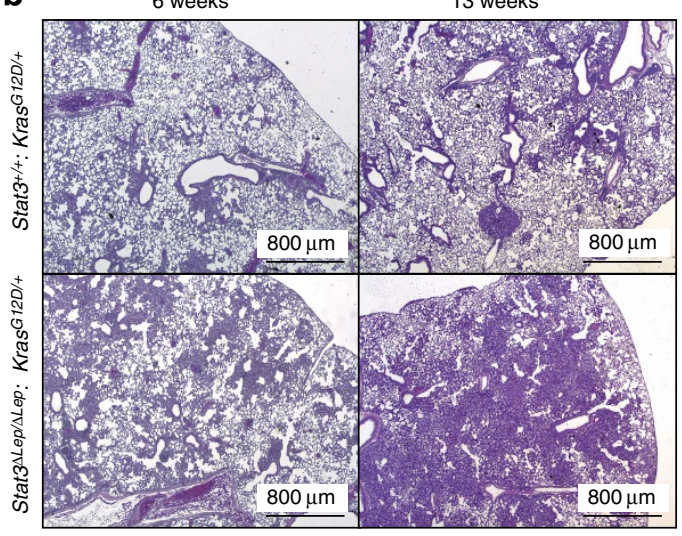

C

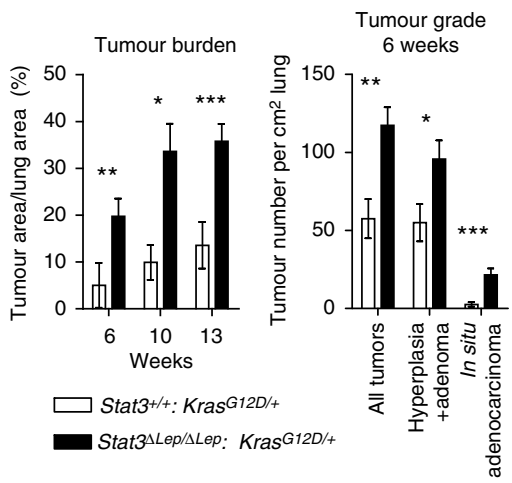

d

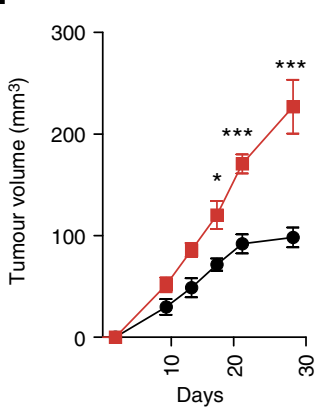

- shControl

- shSTAT3

A549

h

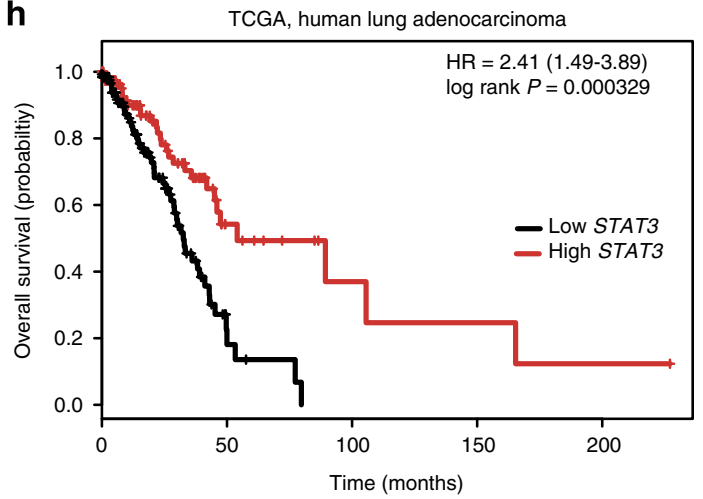

e

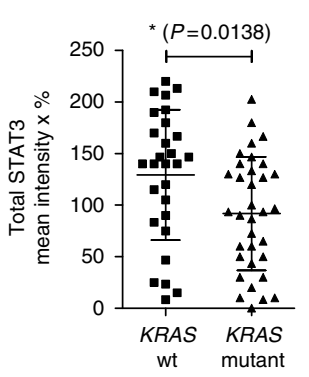

wt mutant
Human adenocarcinomas f

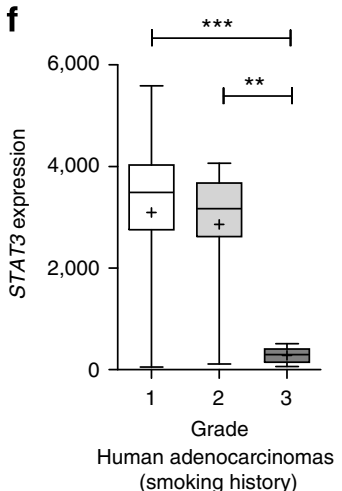

g

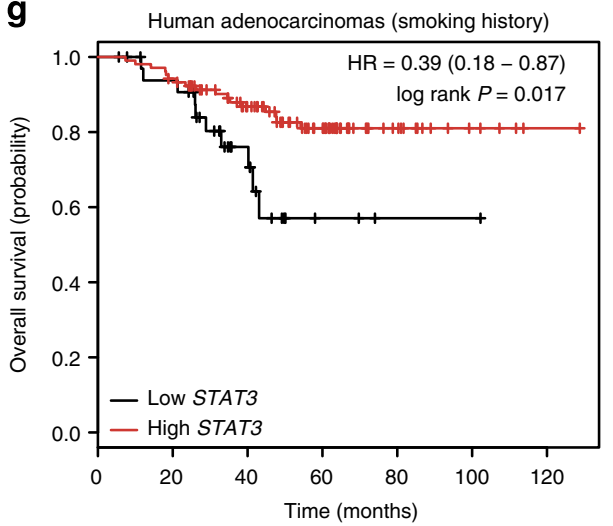

i

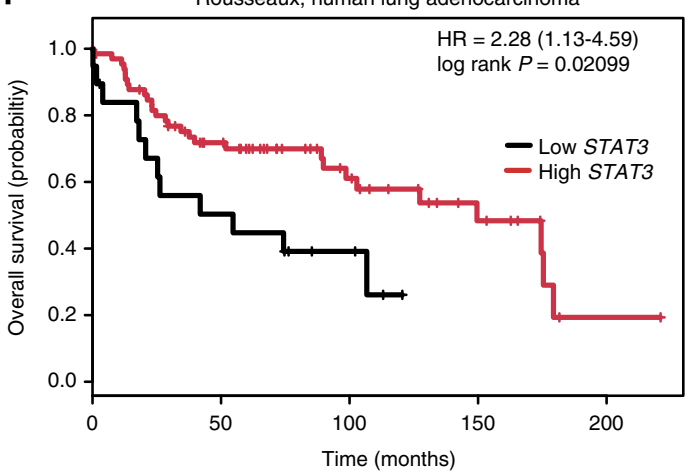

Figure 1 | STAT3 suppresses Kras-induced lung tumorigenesis. (a) Kaplan-Meier plot showing overall survival of male mice with the indicated genotypes infected with AdCre ( $n \geq 10$ male mice per genotype; log-rank test). (b) Representative haematoxylin and eosin stainings and quantification of tumour area/lung area in mice at indicated time points. Data were analysed by Student's $t$-test and are shown as mean \pm s.d., $n=6-10$. Scale bar, $800 \mu m$.

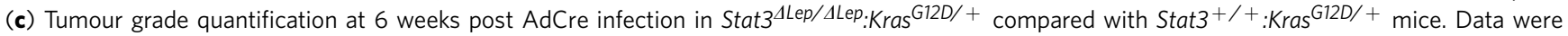
analysed by Student's $t$-test and are shown as mean \pm s.e.m., $n \geq 7$ mice per genotype. (d) Human A549 lung AC cells were transducted with scrambled shRNA (shControl) or shRNA against STAT3 (shSTAT3) and $2 \times 10^{6}$ cells were injected into male nude mice $(n=5$ per group). Data were analysed by two-way analysis of variance with Bonferroni correction and are shown as mean \pm s.e.m. (e) IHC evaluation of total STAT3 in human mucinous adenocarcinomas with or without KRAS mutation. Intensity of staining $(0 /+1 /+2 /+3)$ was multiplied by percentage of stained tumour cells. Data are shown as mean \pm s.d. $n \geq 28$ per group. (Mann-Whitney $U$-test, $P=0.0138$ ) (f) STAT3 mRNA expression of lung adenocarcinoma patients harbouring smoking history at different tumour grades is shown ( $n=139$; grade I, low metastatic potential; grade II, intermediate metastatic potential; grade III, high metastatic potential ${ }^{25}$ ). (Kruskal-Wallis test with Dunn's multiple comparison testing $\left.P<0.0001\right)$ (g) Kaplan-Meier plot showing overall survival of lung adenocarcinoma patients with smoking history stratified by high or low STAT3 mRNA expression (lower quartile, log-rank) $n \geq 35-104$ per group. Kaplan-Meier plot showing overall survival of the (h) TCGA cohort or the (i) Rousseaux cohort stratified by high or low STAT3 mRNA expression (log-rank test). For all graphs: ${ }^{\star} P<0.05 ;{ }^{\star \star} P<0.01 ;{ }^{\star \star \star} P<0.001$.

whether STAT3 signalling is perturbed in human lung ACs and we analysed four different patient cohorts. Interestingly, we found a significant decrease in STAT3 expression levels in KRAS mutant human tumours compared with lung tumours with wild-type KRAS in the first cohort of smoking patients (Fig. 1e). STAT3 activation status was significantly reduced when compared with 
an EGFR mutant cohort (Supplementary Fig. 2b). Furthermore, analysis of the second cohort containing 139 patients with known smoking history showed that STAT3 expression is significantly reduced in grade III tumours (with high metastatic potential ${ }^{25}$; Fig. 1f, $P=<0.0001)^{26}$. Furthermore, in this patient cohort we found that low STAT3 expression levels correlate with reduced overall survival (Fig. 1g, $P=0.017)^{26}$. Of note, it is well established that smoking is strongly associated with KRAS mutations $^{3}$. In this line, STAT3 expression levels do not stratify AC patients without smoking history, a subgroup less prone to harbour KRAS mutations ${ }^{3}$ (Supplementary Fig. 2c). The third and fourth cohort containing 255 and 85 lung AC patient samples, respectively, confirmed our initial findings that STAT3 expression levels are significantly reduced in the high-risk population (Supplementary Fig. 2d and e) ${ }^{27}$. Furthermore, low STAT3 expression correlates with poor overall survival outcome in lung AC patients (Fig. 1h, $P=0.000329$; Fig. 1i, $P=0.002099$ ). Thus, these results indicate that high-grade tumours as well as lung AC are selected for low STAT3 expression presumably to overcome STAT3-mediated tumour suppression, which is consistent with poor survival observed in patients with low levels of STAT3 and a smoking history.

STAT3 alters tumour microenvironment and angiogenesis. Detailed histopathological analysis revealed that Stat3-deficient murine tumours were better vascularized at all investigated time points, which was corroborated by increased $\mathrm{CD} 31^{+}$and von Willebrand Factor staining of vessel walls (Fig. 2a). Tumour angiogenesis is mainly driven by vascular endothelial growth factor alpha (VEGFA) and platelet-derived growth factor alpha (PDGFA) in response to certain stimuli derived from the tumour cells directly or from the surrounding microenvironment ${ }^{28}$. ELISA revealed significantly increased VEGFA levels in lungs from Stat3 $3^{\text {Lep/LLep: Kras }}{ }^{12 D /+}$ mice compared with their littermate controls at 6 weeks, whereas at 13 weeks post AdCre infection no difference could be observed (Fig. 2b). However, at that later time point, $P d g f a$ expression was significantly elevated

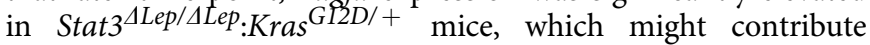
to enhanced tumour vascularization indirectly through the recruitment of angiogenic stroma cells ${ }^{29,30}$ (Fig. 2c).

It has been shown that tumour cell-specific Stat3 blockade leads to changes in the tumour microenvironment and increased immune cell infiltration ${ }^{31}$. Qualitative IHC analysis of the tumour microenvironment revealed that several cell types are present within the lung tumours (NK-cells, T cells, dendritic cells, fibroblasts and macrophages. Supplementary Fig. 3a). As macrophages and granulocytes contribute to tumour angiogenesis $^{32}$, we performed quantitative assessment of these cell types via flow cytometry analysis of bronchoalveolar lavage (BAL) and observed significantly more $\mathrm{CD}_{11 \mathrm{~b}}{ }^{+} \mathrm{Gr} 1^{+}$ granulocytes in Stat $3^{\text {LLep/ALep:Kras }}{ }^{G 12 D /+}$ lungs compared with controls at 6 and 13 weeks post AdCre-induction (Fig. 2d). Furthermore, infiltrating $\mathrm{CD} 11 \mathrm{~b}{ }^{+} \mathrm{F} 4 / 80^{+}$macrophages were sig-

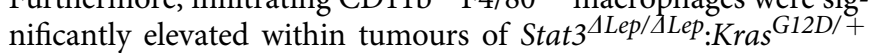
mice at 13 weeks post AdCre in BAL, which was confirmed by IHC (Fig. 2d and Supplementary Fig. 3b). Further analysis of myeloid-derived suppressor cells (MDSCs), which are known to play an important role in suppressing the antitumour immune response ${ }^{33}$, showed that MDSCs from Stat3-deficient mice were more differentiated towards a monocytic phenotype $\left(\mathrm{CD} 11 \mathrm{~b}^{+}\right.$Ly6C $^{\text {high }}$ Ly6G ${ }^{\text {low }}$, M-MDSCs) than to a granulocytic phenotype (G-MDSCs, CD11b ${ }^{+}$Ly6 $\mathrm{C}^{\text {low }}$ Ly6 $6 \mathrm{G}^{\text {high }}$ ) at later stages of tumour development (13 weeks; Fig. 2e). M-MDSCs have been postulated to be more potent in suppressing T-cell responses 33 . In line with this, we found that Stat $3^{\text {SLep/ALep: Kras }}{ }^{G 12 D /+}$ animals showed an increased $\mathrm{CD} 4{ }^{+} / \mathrm{CD} 8^{+}$ratio compared with control mice at the latest time point (13 weeks), indicating a suppression of the cytotoxic $\mathrm{CD}^{+} \mathrm{T}$ cells response within the tumours (Fig. 2f).

We further investigated tumour microenvironment and angiogenesis within our patient cohort. Correlations between CD31 or VEGF and P-STAT3 or STAT3 did not reveal any significant differences between KRAS wild-type and mutant cases (Supplementary Fig. 3c,d). Qualitative assessment of infiltrating immune cells revealed that only $3 \%$ of tumours were infiltrated with macrophages and we did not observe any differences between both genotypes (Supplementary Fig. 3e). However, the number of tumours scoring positive for infiltrating granulocytes was higher in KRAS mutant compared with KRAS wild-type tumours (18\% within the KRAS mutant versus $10 \%$ within KRAS wild-type cohort); in addition, less cases of KRAS mutant tumours scored positive for lymphocyte infiltration compared with KRAS wild-type tumours (31\% within the KRAS mutant versus $48 \%$ in KRAS wild-type cohort; Supplementary Fig. 3e).

In order to substantiate the findings of our mouse model, we analysed the human AC xenograft samples and found that tumours lacking STAT3 showed significantly increased numbers of infiltrating $\mathrm{CD}_{11 \mathrm{~b}}{ }^{+} \mathrm{Gr} 1^{+}$and $\mathrm{CD}^{+} 1 \mathrm{~b}^{+} \mathrm{F} 4 / 80^{+}$cells (Fig. 2g) and were significantly more vascularized (Fig. 2h), thus confirming our observations in the murine model. Together, these results implicate that STAT3 ablation results in a considerably different tumour microenvironment composed of more myeloid cells, increased $\mathrm{CD} 4{ }^{+} / \mathrm{CD} 8^{+}$lymphocyte ratio and enhanced angiogenesis in our murine as well as in the human AC xenograft models.

STAT3 regulates chemoattractive CXCL1 expression. To test if the increased recruitment of myeloid cells is a result of changes within the local cytokine and growth factor milieu in Stat $3^{\text {LLep/LLep }: K r a s}{ }^{G 12 D /+}$ tumours, we performed ELISA of several key cytokines, which may modulate the infiltration of myeloid cells into tumours. IL-2, IL-12p40, IL-4 and IL-17 were not detectable and we did not find differences in IL-5, interferon- $\gamma$, IL-10, IL-1 $\alpha$, EGF, OSM expression between the two genotypes (Supplementary Figs $4 \mathrm{a}$ and 1b). Granulocytemacrophage colony-stimulating factor and IL-6 expression were upregulated in Stat3-deficient tumours only at the latest time point (Supplementary Figs 4a and 1b). Interestingly, CXCL1 was the only cytokine, which showed a significant increase in lungs from Stat ${ }^{\text {LLep/LLep }: K r a s}{ }^{G 12 D /+}$ mice compared with their control littermates at all time points (Fig. 3a). Cxcll (murine orthologue to human CXCL1 and $I L-8$ (ref. 34)) is a well-known and potent chemo-attractant for macrophages and granulocytes and can induce angiogenesis ${ }^{35,36}$. In situ hybridization not only corroborated the increased expression of Cxcl1 in Stat3-deficient tumours but also identified the tumour cells as the main cellular origin of Cxcl1 as confirmed by staining for the alveolar type 2 cell-specific marker SP-C (surfactant protein $\mathrm{C}$ ) in serial sections (Fig. 3b and Supplementary Fig. 4b). Together, these data suggest that STAT3 negatively regulates the angiogenic and proinflammatory cytokine CXCL1 in the murine model.

Next, we analysed the patient cohorts for $I L-8$ expression, the human orthologue of Cxcl1 (ref. 34). Interestingly, STAT3 and IL-8 expression tended to inversely correlate in KRAS mutant samples, whereas KRAS wild-type samples showed a trend towards a positive correlation between STAT3 and IL-8 expression (Supplementary Fig. 4c). Furthermore, high IL-8 expression levels have been associated with poor prognosis in smoking patients ${ }^{37}$, a finding which we could confirm within the second patient cohort used in this study (Fig. $3 \mathrm{c})^{26}$. 
a

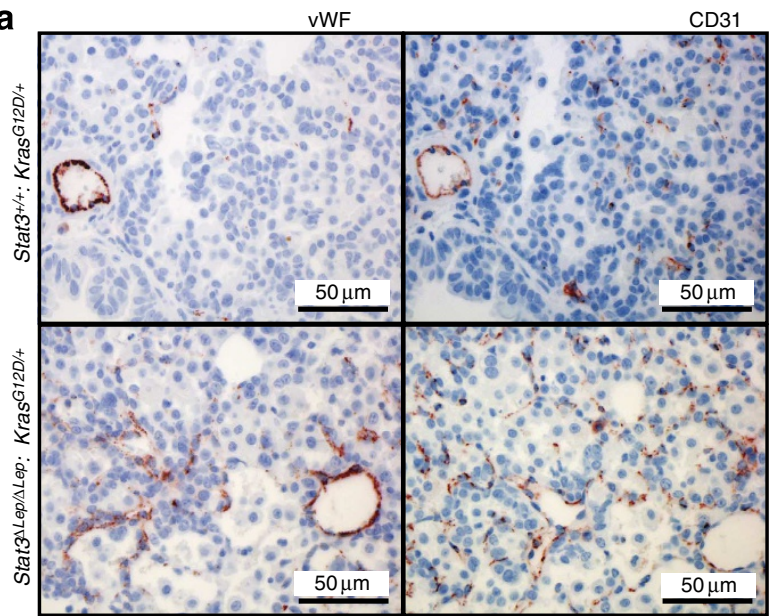

b

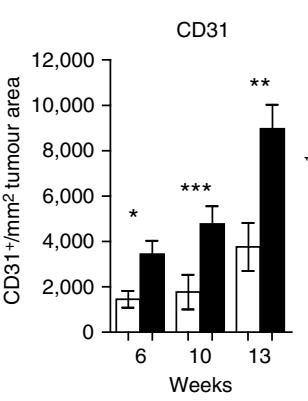

$\square$ Stat3 $^{+/+}:$Kras ${ }^{G 12 D /+}$

Stat3LLep/LLep: KrasG12D/+

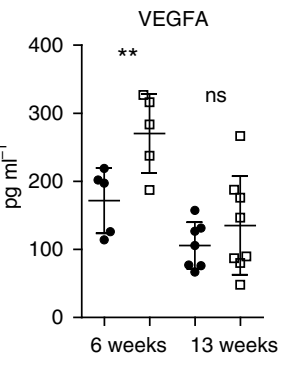

- Stat3+/+: KrasG12D/+

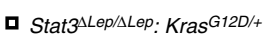

C

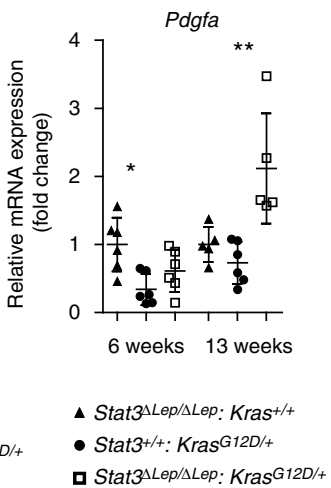

d

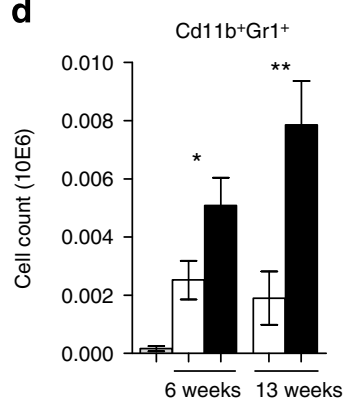

e

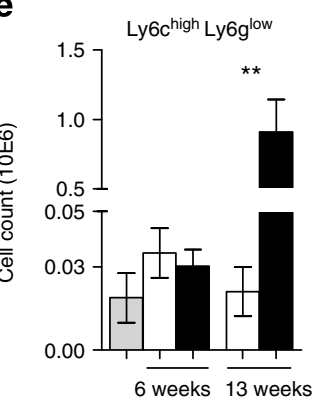

f

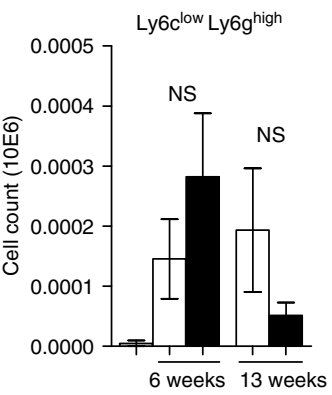

$\mathrm{CD} 4+$ /CD8+ratio

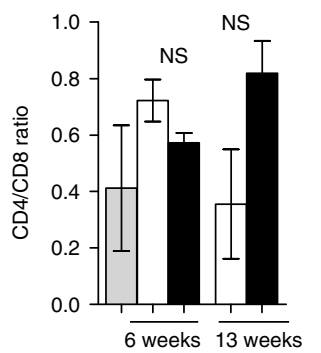

\footnotetext{
$\square$ Stat3 $^{+/+}: \mathrm{Kras}^{+/+}$

Stat3+/+: KrasG12D/t

Stat3دLep/LLep: Kras G12D/+
}

$6 \overline{\text { weeks }} \overline{13 \text { weeks }}$

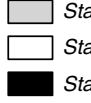

$\mathrm{Stat}^{+/+}: \mathrm{Kras}^{+/+}$

Stat3+/+: KrasG12D/+

Stat3ALep/LLep: KrasG12D/+

g

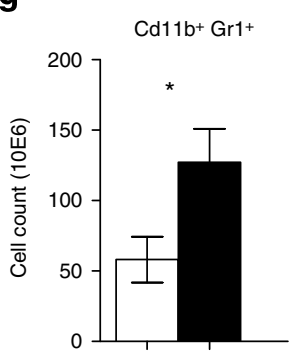

h

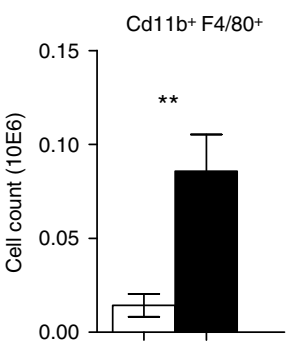

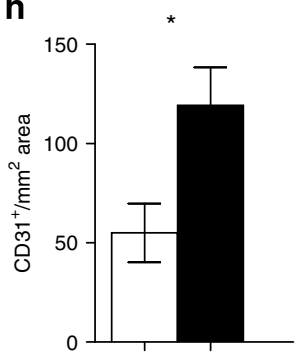

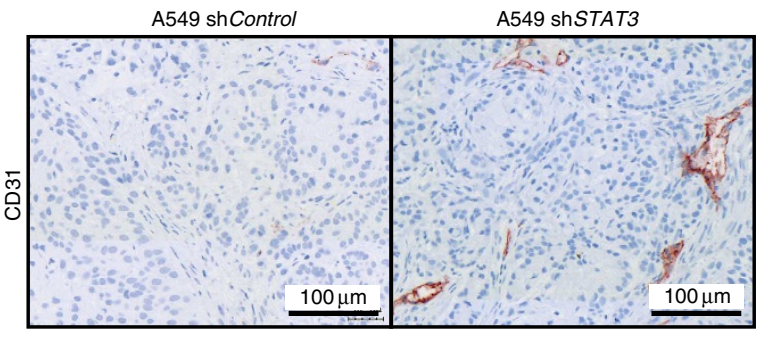

A549 shControl

A549 shSTAT3

Figure 2 | STAT3 alters tumour microenvironment and angiogenesis. (a) IHC analysis of von Willebrand Factor (vWF) and CD31 is shown in consecutive sections of murine lung tumours of the indicated genotype. On right, $\mathrm{CD} 31^{+}$counts per tumour area $\left(\mathrm{mm}^{2}\right)$ quantification at indicated time points. Data were analysed by Student's $t$-test and displayed as mean \pm s.e.m. $n \geq 5$ tumours per mouse with $n \geq 4$ mice per genotype and time point. Scale bar, $50 \mu \mathrm{m}$. (b) ELISA of VEGFA levels in lung tumour lysates at indicated time points. Data were analysed by Student's $t$-test and displayed as mean \pm s.d., $n \geq 6$ mice per genotype and time point. (c) Expression levels of Pdgfa in total lungs were measured by quantitative real-time PCR at indicated time points. Values are presented as fold change of relative mRNA expression compared with Stat $3^{\Delta L e p / \Delta L e p: K r a s}{ }^{+} /+$mice. Data were analysed by one-way analysis of variance (ANOVA) with Tukey's multiple comparison test and are shown as mean \pm s.d., $n \geq 5$ animals/genotype. (d) Flow cytometric analysis of $\mathrm{Cd}_{11 \mathrm{~b}}{ }^{+} \mathrm{Gr}^{+}$granulocytes and $\mathrm{Cd11b}{ }^{+} \mathrm{F} 4 / 80^{+}$macrophages in bronchoalveolar lavage (BAL) at 6 and 13 weeks post AdCre. Data were analysed by one-way ANOVA with Tukey's multiple comparison test and are shown as mean \pm s.e.m. (e) Flow cytometric analysis of myeloid-derived suppressor cell (MDSC) subsets at 6 and 13 weeks post AdCre. Data were analysed by one-way ANOVA with Tukey's multiple comparison test and are shown as mean \pm s.e.m. (f) Ratio of $\mathrm{CD} 4^{+} / \mathrm{CD} 8{ }^{+} \mathrm{T}$-cell counts in BAL are shown. Data were analysed by Kruskal-Wallis test with Dunn's multiple comparison testing and are shown as mean \pm s.e.m. Data displayed in $\mathbf{d}-\mathbf{f}$ are $n \geq 6$ mice per genotype and time point, 13-week group represents two independent experiments. (g) Flow cytometric analysis of $\mathrm{Cd11b}+\mathrm{Gr}^{+}$and $\mathrm{Cd11b}{ }^{+} \mathrm{F} 4 / 80^{+}$cells of A549 shControl versus A549-shSTAT3 xenograft tumours. Data were analysed by Student's $t$-test and are displayed as mean \pm s.e.m. ( $n \geq 8$ tumours; $\geq 4$ mice per group). (h) IHC analysis and representative pictures of CD31 ${ }^{+}$counts per xenograft tumour area $\left(\mathrm{mm}^{2}\right)$. Scale bar, $100 \mu \mathrm{m}(n=8$ tumours $/ \geq 4$ mice). Data were analysed by Student's $t$-test and are shown as mean \pm s.e.m. For all graphs: ${ }^{\star} P<0.05 ;{ }^{\star \star} P<0.01 ;{ }^{* \star} P<0.001$. 
a

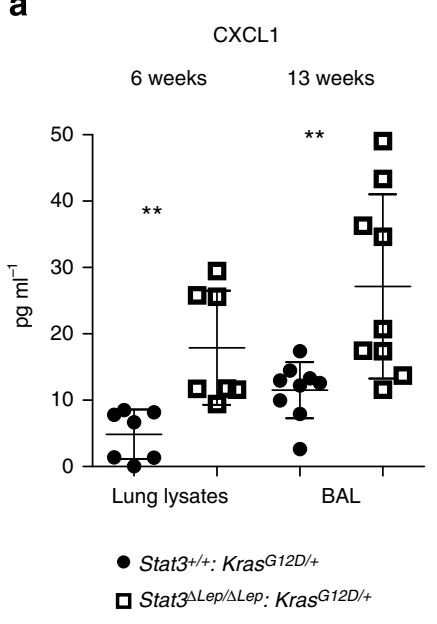

b
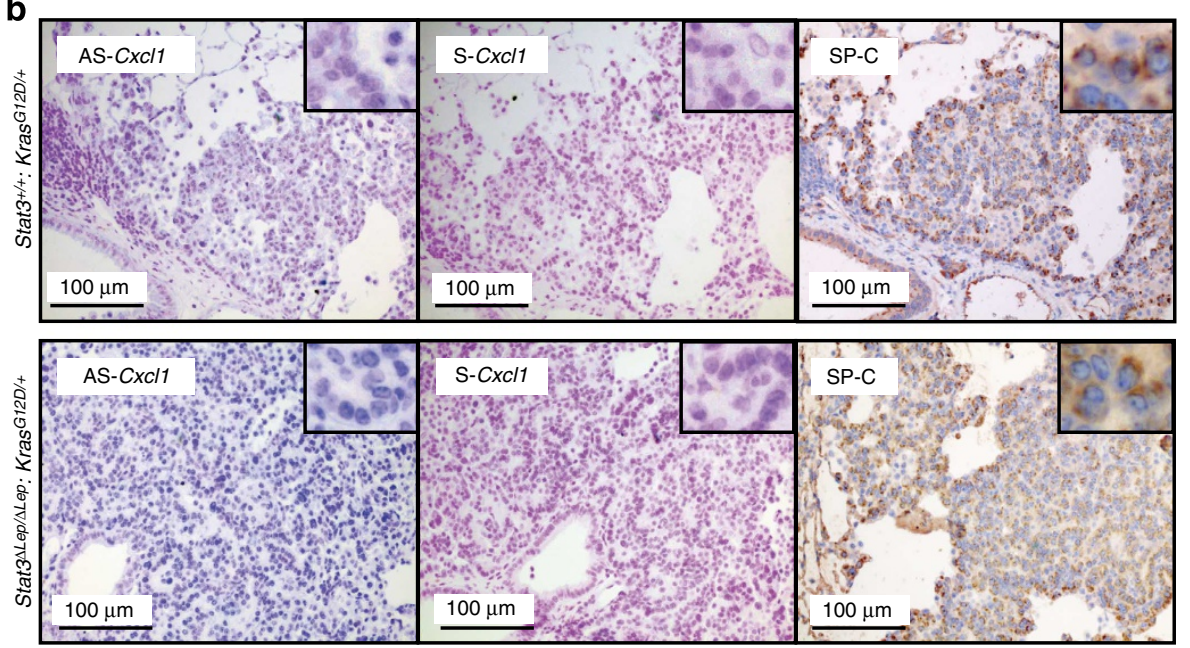

C

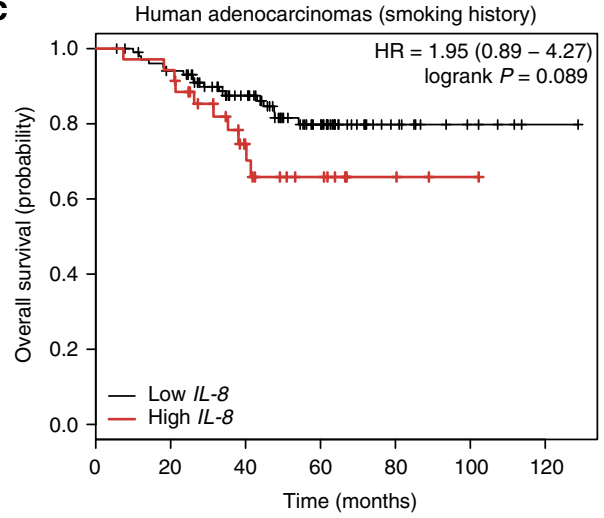

d

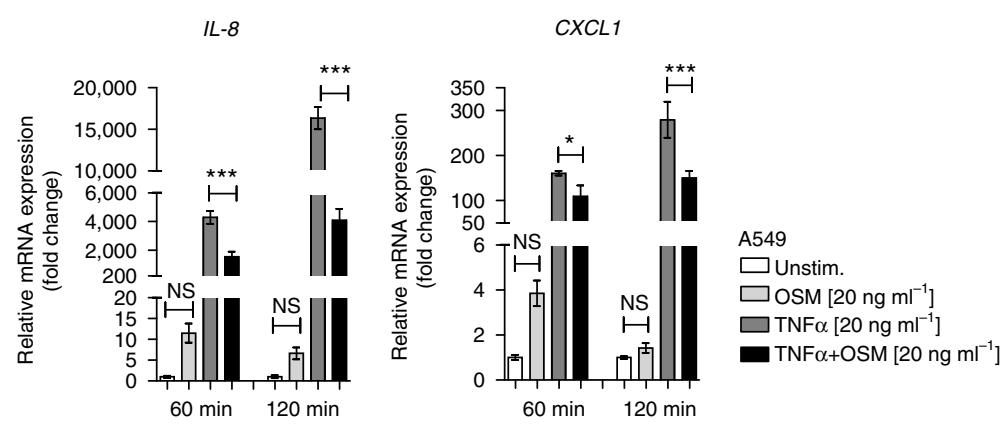

e

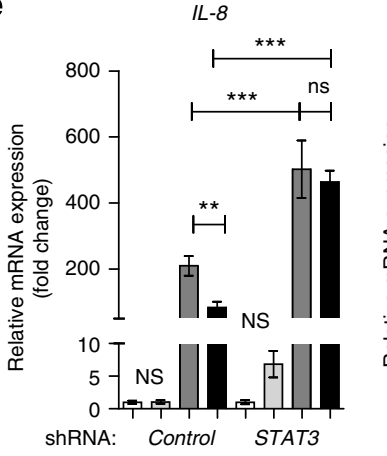

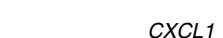

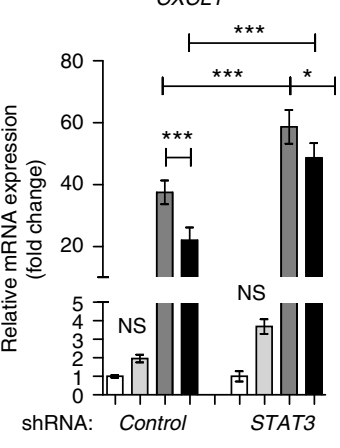

A549

$\square$ Unstim.

$\square$ OSM [20 $\left.\mathrm{ng} \mathrm{ml}^{-1}\right] 60 \mathrm{~min}$

$\square \mathrm{TNF} \alpha\left[20 \mathrm{ng} \mathrm{ml}^{-1}\right] 60 \mathrm{~min}$

TNF $\alpha+O S M\left[20 \mathrm{ng} \mathrm{ml}^{-1}\right] 60 \mathrm{~min}$ f

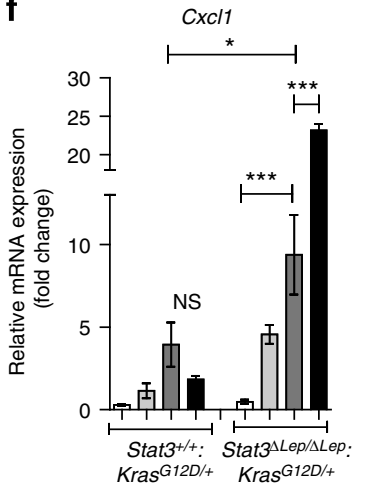

Primary pneumocytes

$\square$ Unstim.

$\square$ OSM [20 $\left.\mathrm{ng} \mathrm{ml}^{-1}\right] 60 \mathrm{~min}$

$\square \mathrm{TNF} \alpha\left[20 \mathrm{ng} \mathrm{ml}^{-1}\right] 60 \mathrm{~min}$

TNF $\alpha+O S M\left[20 \mathrm{ng} \mathrm{ml}^{-1}\right.$ ] $60 \mathrm{~min}$ g $\quad C x c 11$

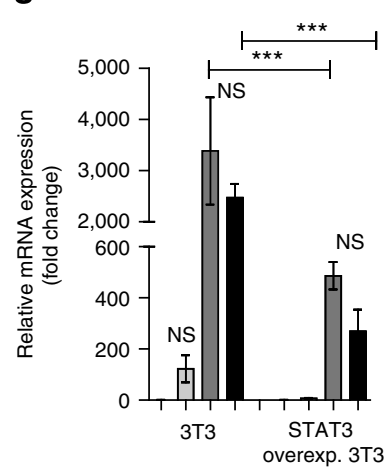

Murine fibroblasts

$\square$ Unstim.

$\square$ OSM $\left[20 \mathrm{ng} \mathrm{ml}^{-1}\right] 60 \mathrm{~min}$ $\square$ TNF $\alpha\left[20 \mathrm{ng} \mathrm{ml}^{-1}\right] 60 \mathrm{~min}$ TNF $\alpha+O S M\left[20 \mathrm{ng} \mathrm{ml}^{-1}\right] 60 \mathrm{~min}$

Figure 3 | STAT3 regulates chemoattractive CXCL1 expression. (a) ELISA of CXCL1 levels in lung lysates and BAL at 6 and 13 weeks post AdCre. Data were analysed by Student's $t$-test and shown as mean \pm s.d., $n \geq 7$ mice per genotype and time point. (b) Representative in situ hybridization with probes specific for murine $\mathrm{Cxcl1}$ (AS) in lung tumours at 13 weeks post AdCre. Sense probes (S) were used as negative control and surfactant protein $\mathrm{C}$ (SP-C) as lung epithelial maker. Scale bar, $100 \mu \mathrm{m}$. (c) Kaplan-Meier plot showing overall survival of lung adenocarcinoma patient samples harbouring smoking history with high or low IL-8 mRNA expression (expression values of upper quartile, log-rank test) $n \geq 35-104$ per group. (d) Human A549 lung cancer cell line (KRAS G12S mutated) was stimulated with designated cytokines for indicated time points. IL-8 or CXCL1 expression was analysed via qRT-PCR. Data were analysed by one-way ANOVA with Tukey's multiple comparison test and shown as mean \pm s.d. (e) A549 cells transducted with lentiviral vectors expressing nonspecific scrambled small hairpin (sh)RNA or shRNA against STAT3 were stimulated with indicated cytokines for 60 min. Data were analysed by one-way ANOVA with Tukey's multiple comparison test and shown as mean \pm s.d. (f) Primary pneumocytes were isolated and infected with AdCre. $120 \mathrm{~h}$ post infection cells were stimulated with indicated cytokines for $60 \mathrm{~min}$. Cxcl1 expression was analysed by qRT-PCR. Data are shown as mean \pm s.e.m. (g) Murine 3 T3 fibroblasts and STAT3 overexpressing 3T3 fibroblasts were stimulated as in f. Cxcl7 expression was analysed by qRT-PCR. Data were analysed by one-way ANOVA with Tukey's multiple comparison test and shown as mean \pm s.d. Values in $\mathbf{d}-\mathbf{g}$ are presented as fold change of relative mRNA expression compared with each unstimulated individual cell line. At least two independent experiments with three individual plates per stimulation were performed. For all graphs: ${ }^{\star} P<0.05 ;{ }^{\star \star} P<0.01 ;{ }^{\star \star \star} P<0.001$. 
We next performed various cytokine stimulation experiments in vitro to corroborate the Stat3-mediated repression of Cxcll observed in the murine model. First, we confirmed that the human KRAS mutant AC cell line A549 activates STAT3 in response to cytokines such as OSM, IL-6 and EGF found to be expressed in our murine lung tumours in vivo (Supplementary Figs $1 \mathrm{~b}$ and $4 \mathrm{~d})$. We used tumour necrosis factor- $\alpha$ (TNF $\alpha$ ) to stimulate NF- $\mathrm{KB}$ induced $I L-8$ and CXCL1 expression in A549 cells and analysed the effect of co-stimulating STAT3 by OSM or IL-6. As expected, TNF $\alpha$ stimulation induced a marked $I L-8$ and CXCL1 expression, which was significantly inhibited by cotreatment with OSM (Fig. 3d). Interestingly, OSM treatment alone also led to a very small and transient increase of $I L-8$ and CXCL1. Similar results were obtained with IL-6 co-stimulation (Supplementary Fig. 4e). Importantly, small hairpin-mediated knockdown of STAT3 confirmed that the observed effects on CXCL1 and $I L-8$ repression were at least in part mediated by STAT3 (Fig. 3e and Supplementary Fig. 4f).

Next, we isolated primary mouse alveolar type 2 pneumocytes from the lungs of Stat $3^{\text {flox } / \text { flox }}:$ Kras $^{\mathrm{LSL}-G 12 \mathrm{D} /+}$ and Stat $3^{+1+}$ : Kras ${ }^{\mathrm{LSL}-\mathrm{G} 12 \mathrm{D} /+}$ animals (Supplementary Fig. 4g). Complete deletion of STAT3 was observed $120 \mathrm{~h}$ post AdCre infection in vitro (Supplementary Fig. $4 \mathrm{~h}$ ). In these primary cells, TNF $\alpha$ treatment again triggered $\mathrm{Cxcl} 1$ expression albeit less pronounced compared with A549 cells (Fig. 3f). Importantly, Stat3-deficient Kras ${ }^{\mathrm{G} 12 \mathrm{D} /+}$-mutant pneumocytes expressed significantly increased levels of Cxcl1 upon TNF $\alpha$ stimulation compared with syngeneic Stat3-proficient cells. Upon co-stimulation with OSM, Stat3-deficient pneumocytes not only failed to repress Cxcl1 expression but even showed vastly increased Cxcl1 expression levels (Fig. 3f). Conversely, experiments using murine 3T3 fibroblasts overexpressing STAT3 (ref. 10) showed that STAT3 is not only required but is sufficient to repress Cxcl1 expression (Fig. 3g). These results suggest that STAT3 represses Cxcll expression in vivo and in vitro.

CXCL1 inhibiton reverts oncogenic effects of STAT3 ablation. Next, we tested whether CXCL1 is responsible for the increased tumour growth supported by vascularization and infiltration. We used the SB225002 compound to inhibit CXCL1's cognate receptor CXCR2, which we found to be expressed on epithelial, endothelial and myeloid cells within the lung tumours (Supplementary Fig. 5a) ${ }^{36,38}$. CXCR2 antagonist treatment was conducted in two ways: first, we treated the mice 1 week after AdCre tumour induction (Supplementary Fig. 5b). After 5 weeks of continuous CXCR2 blockade, we could not only observe a marked reduction in tumour vascularization but importantly tumour incidence and overall tumour burden were reduced, suggesting that CXCL1 is crucial for tumour development at early stages in Stat ${ }^{4 L e p / \Delta L e p}: K_{r a s}{ }^{G 12 D /}+$ mice (Fig. $4 \mathrm{a}$,b). Second, we tried to mimic a therapeutic setting and treated tumour-bearing mice (Supplementary Fig. 5b). After 7 weeks of treatment, we could observe a significant reduction of vascularization and of infiltrating $\mathrm{F} 4 / 80^{+}$macrophages in the tumours of Stat $3^{\text {LLep/ALep }}$ : Kras ${ }^{\mathrm{Gl} D \mathrm{D}}+$ mice (Supplementary Fig. 5c), but not in overall tumour burden (Supplementary Fig. 5d). To further confirm these findings in a human cell line, we performed a small hairpinmediated double knockdown of IL-8 and STAT3 in A549 cells (Supplementary Fig. 5e). Indeed, enhanced tumour growth of shSTAT3 cells was significantly reduced upon knockdown of $I L-8$ (Fig. 4c). Furthermore, vascularization as well as macrophage and granulocyte infiltration was reduced in the $I L-8 / S T A T 3$ double knockdown tumours compared with STAT3 knockdown tumours (Fig. $4 \mathrm{~d}, \mathrm{e})$. Interestingly, knockdown of $I L-8$ alone showed an increased tumour growth in nude mice compared with shControl cells for unknown reasons (Fig. 4c). Taken together, these data suggest that loss of STAT3 in the tumour cells results in increased CXCL1/IL-8 expression triggering infiltration of myeloid cells as well as augmented vascularization, and identifies CXCL1/IL-8 as an essential mediator for the accelerated development and progression of Stat3-deficient tumours.

STAT3 retains p65 in the cytoplasm to reduce NF- $\mathrm{B}$ activity. Having demonstrated the importance of STAT3-mediated repression of CXCL1 in tumour cells in vitro and in vivo, we set out to mechanistically interrogate this pathway further. As NF- $\kappa \mathrm{B}$ subunit p65 is an important regulator of CXCL1 expression, we first examined the p65 activation ${ }^{39,40}$. Increased levels of activated p65 were observed, which was mirrored by increased expression

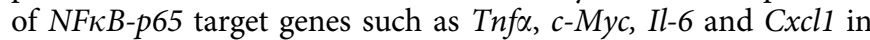
Stat3-deficient lungs, whereas Bcl2, Bcl2l1, Ccnd1 and Ccl5 were comparably expressed (Figs 3a,5a,b; Supplementary Figs $1 \mathrm{~b}$ and $6 \mathrm{~b})^{41}$. These data demonstrate that Stat3-deficient tumours show increased $N F-\kappa B$ activity.

We examined the association of $N F-\kappa B$ and STAT3 expression in our patient sample archive. We found a significant positive correlation of $N F-\kappa B$ and STAT3 expression in KRAS wild-type samples. On the contrary, we found a trend towards an inverse correlation between $N F-\kappa B$ and STAT3 expression in KRAS mutant samples (Supplementary Fig. 6c).

As STAT3 has been shown to compete with NF- $\kappa \mathrm{B}$ on various promoters ${ }^{42,43}$, we further analysed the crosstalk of STAT3 and NF- $\kappa \mathrm{B}$ binding on the human CXCL1 and the mouse Cxcl1 promoter. Within the mouse Cxcl1 promoter, we found several putative STAT3-binding sites-two of them overlapping with putative NF- $\kappa \mathrm{B}$-binding sites and conserved in humans (hereafter termed responsive element, RE1 and 2; Supplementary Fig. 6d). However, electrophoretic mobility shift assays of all putative binding sites (Supplementary Fig. 6e) as well as in vivo chromatin immunoprecipitation experiments could not reveal strong endogenous binding of STAT3 on RE1 or RE2 (Supplementary Fig. 6f). We next tested the human A549 cell line focusing on the RE2 site (Fig. 5c). Upon TNF $\alpha$ treatment, we could detect efficient recruitment of p65 to the CXCL1 promoter and co-stimulation of STAT3 signalling by OSM treatment indeed markedly reduced p65 recruitment. However, we failed to detect direct STAT3 binding at the CXCL1 promoter site (Fig. 5c), indicating that STAT3 regulates p65-induced CXCL1 expression by means other than competing for promoter-binding sites.

As STAT3 and the NF- $\kappa \mathrm{B}$ subunit $\mathrm{p} 65$ have been reported to interact at the protein level ${ }^{43-45}$, we next tested if STAT3 may bind and control subcellular localization of p65. Coimmunoprecipitation experiments in A549 cells showed that the majority of STAT3 and NF- $\mathrm{BB}-\mathrm{p} 65$ interact in the cytoplasm under basal conditions as well as upon stimulation with OSM, TNF $\alpha$ or a combination thereof (Fig. 5d). We next quantified nuclear p65 levels normalized to poly (ADP-ribose) polymerase family, member 1 (PARP). Upon OSM stimulation, a reduction of nuclear p65 compared with unstimulated conditions was observed. Increased levels of nuclear p65 were obtained upon TNF $\alpha$ stimulation, which was reduced to basal levels upon costimulation with OSM (Fig. 5d). To further confirm this, we analysed the subcellular localization of p65 using immunofluorescence imaging of STAT3-proficient A549 cells and compared it to the STAT3 knockdown cell line (Fig. 5e). Under basal conditions, A549-shSTAT3 cells already showed increased levels of nuclear p65 compared with controls. Interestingly, we observed a reduction of nuclear p65 in OSMstimulated A549-shSTAT3 cells compared with unstimulated controls, indicating that additional OSM-induced effectors other 
than STAT3 are also regulating the cytoplasmic-nuclear trafficking of p65. As expected, both cell lines showed increased levels of nuclear p65 upon TNF $\alpha$ stimulation. Importantly, co-stimulation with OSM resulted in a significant reduction of TNF $\alpha$-induced nuclear p65 accumulation in A549 scrambled control cells but not in shSTAT3-infected cells (Fig. 5e). In summary, our results suggest that STAT3 represses NF-KB- dependent CXCL1 expression by sequestering NF- $\kappa \mathrm{B}$ in the cytoplasm and mechanistically delineate a novel tumoursuppressive pathway governed by the STAT3-NF- $\mathrm{KB}-\mathrm{CXCL} 1$ axis.

\section{Discussion}

Personalized treatment decisions based on the genetics of the individual tumour will be paramount to combat malignancies in a

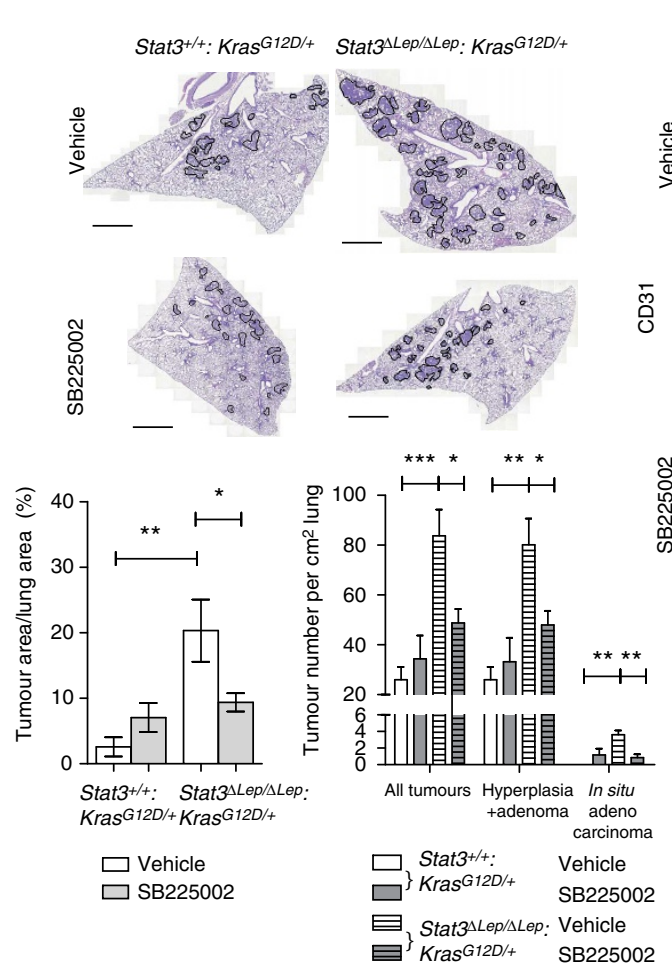

C

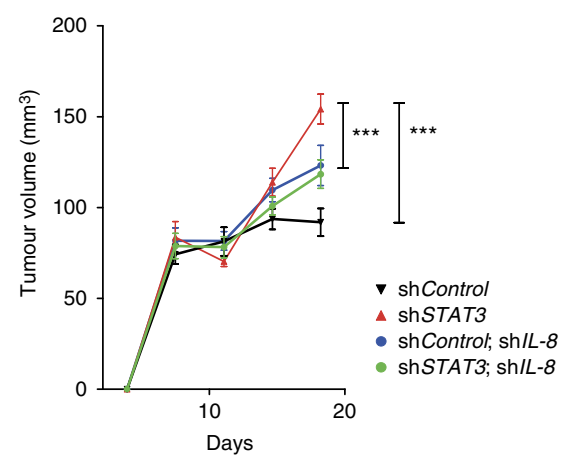

e

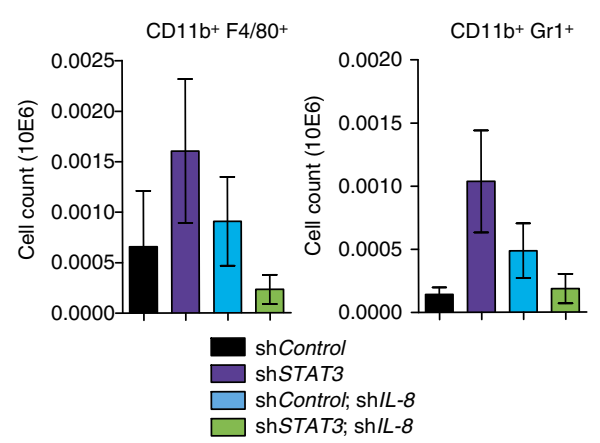

b

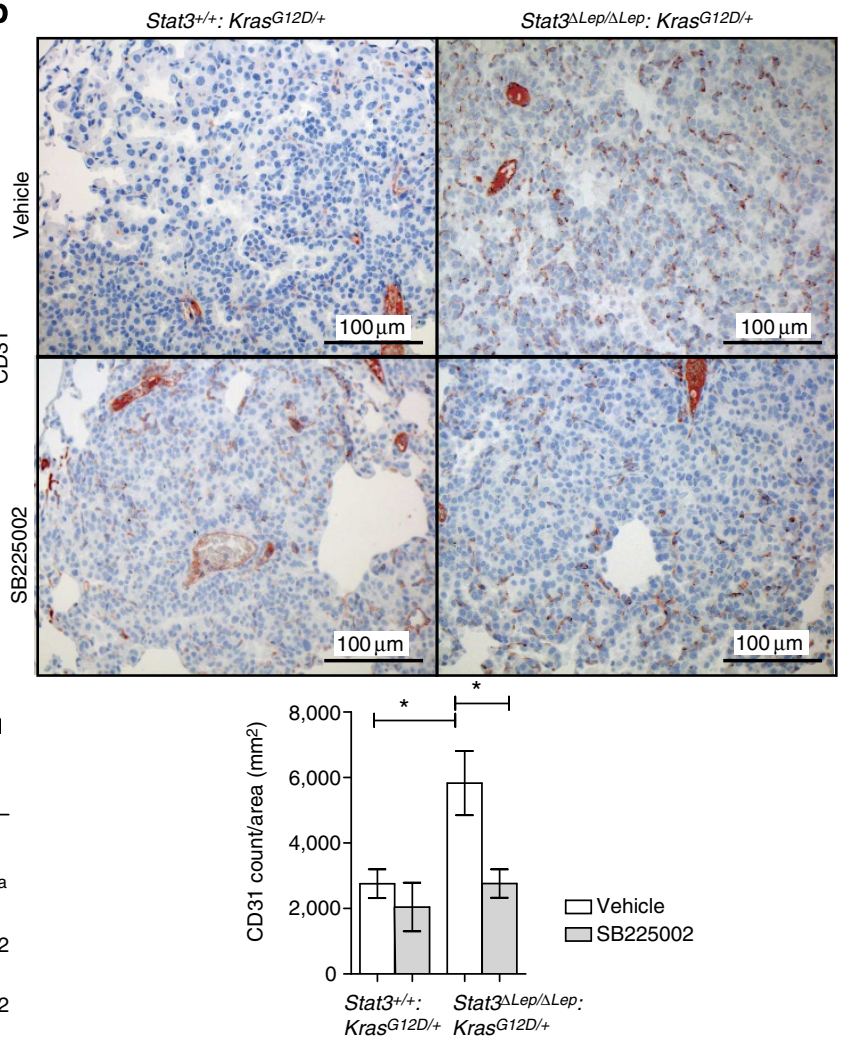

d
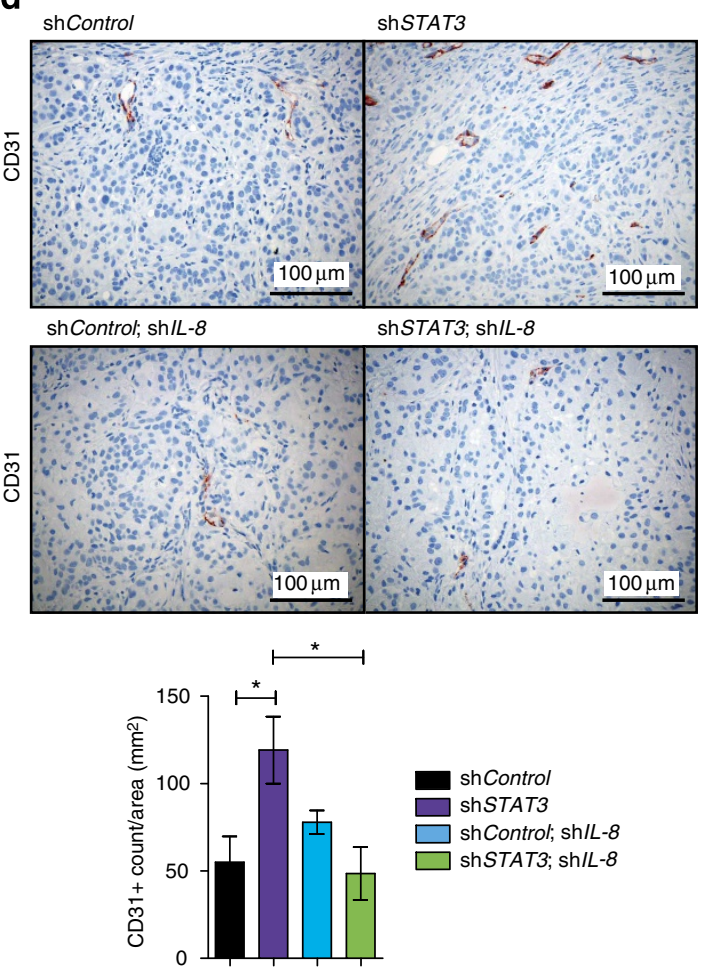
the near future. STAT3 has been implicated in several malignancies $5,7,13,14,46$ and therefore, clinical studies are currently evaluating the efficacy of STAT3 inhibition in various kinds of cancer ${ }^{46}$. However, although STAT3 traditionally has been described as an oncogene ${ }^{7,9}$, recent reports have shown that STAT3 can also behave as a tumour suppressor in the very same organ systems $s^{9,11,12,42}$. In addition, other reports validated high STAT3 expression as a potential marker for good prognosis in human colorectal carcinoma and breast cancer ${ }^{47,48}$. Therefore, the idea emerged that STAT3 function is context-dependent either with regards to the oncogenic driver mutation, cancer type or the specific tumour microenvironment.

Specifically in lung cancer, STAT3 has been shown to function as one of the main downstream transcription factors in EGFR mutant $\mathrm{ACs}^{13}$ and to act as an oncogene in a chemically induced mouse model of lung cancer ${ }^{49}$. In addition, several studies of human lung cancer specimens have shown the activation of STAT3 in those tumours ${ }^{13,14,16}$. These findings made STAT3 an attractive drug target to treat NSCLC patients. We hypothesized that lung AC patients, especially those with activating KRAS mutations, might also benefit from STAT3 inhibition. As KRAS is still considered as an undruggable target and is responsible for $30 \%$ of all Caucasian AC cases, we investigated whether STAT3 inhibition has a potential survival benefit in a preclinical lung cancer mouse model and in a human cell line xenograft model. However, our results clearly show that STAT3 behaves as a haploinsufficient tumour suppressor during $\mathrm{Kras}{ }^{G 12 D_{\text {-induced }}}$ lung tumorigenesis. Mice lacking Stat3 signalling specifically within Kras ${ }^{G 12 D}$-mutant tumour cells not only display increased tumour initiation and tumour cell proliferation but also accelerated malignant progression and ultimately markedly reduced survival compared to mice with intact Stat 3 signalling. We could confirm these observations in human lung AC cellderived xenografts. Of note, others have shown that reducing STAT3 activity by using STAT3 inhibitors or STAT3 decoy oligonucleotides suppressed tumour growth in xenograft-derived NSCLC cell lines ${ }^{18,50,51}$. However, by using a powerful short hairpin RNA (shRNA)-mediated loss-of-function approach, we found that STAT3 suppresses tumorigenesis in A549 cell-derived xenografts, thus supporting our findings in the murine model. Despite the fact that Stat3-deficient tumours had a growth advantage, they did not progress to become more invasive than Stat3-proficient tumours or metastatic. Tumour invasion and metastasis are a multistage development in which, in addition to neo-angiogenesis, malignant tumour cells have to undergo EMT, detach from the primary tumour, migrate and pass mechanical barriers. This is a complex process driven by multiple somatic aberrations. Thus, most likely, Stat3-deficient tumours need additional hits to become invasive and to metastasize.
Interestingly, the observed survival disadvantage is more pronounced in male mice than in female mice reminiscent of observations made in the clinic: although women have a higher incidence rate in developing lung cancer, they have a better survival outcome compared with men ${ }^{52}$. Further on, STAT3 already has been linked to gender-specific diseases in liver and lung cancer ${ }^{53,54}$. Detailed analysis of this gender-specific difference associated with STAT3 will be of high interest. One of the main pro-inflammatory and neo-angiogenesis promoting cytokines responsible for our observations in Stat3-deficient tumours is Cxcl1. Cxcl1, the murine orthologue to human $I L-8$ and CXCL1, is known to be RAS dependent ${ }^{40}$, to directly attract myeloid cells and to stimulate endothelial cells to promote angiogenesis ${ }^{35,36}$. Clinically, IL-8 expression is associated with a tendency for poor prognosis in human lung cancer ${ }^{37}$ and expression of CXCR2 also correlates with smoking and poor prognosis $^{55}$. In agreement with this, we could confirm that $I L-8$ expression tends to correlate with poor survival in smoking patients (who frequently acquire KRAS mutations) within the publically available AC patient cohort used in this study. Interestingly, we could also show that low STAT3 expression has a tendency to correlate with increased $I L-8$ mRNA expression in KRAS mutant patient samples.

To investigate whether CXCL1 is the central angiogenesis and tumour-promoting factor in our model and a potential therapeutic target, we treated the mice with SB225002, a smallmolecule antagonist of CXCR2. Treating Stat3-deficient mice at tumour initiation resulted in a reduction of tumour growth as well as a decrease in vascularization compared with vehicletreated control mice. This result corroborates that CXCL1 signalling indeed accelerates tumour growth at early stages. In addition, we cannot rule out that CXCL1 also acts as an autocrine tumour-promoting factor in lung cancer cells ${ }^{56-58}$. We also tried to validate CXCL1 as a therapeutic target by blocking CXCL1 signalling in established tumours. By using this approach, we could show that tumour vascularization and infiltration of macrophages were reduced at the end stage of the treatment; however, we did not observe any effect on tumour growth. This result indicates that other effectors in Stat3-deficient tumours, like elevated NF- $\mathrm{\kappa B}$ activity and/or increased $c-M y c$ expression, may contribute to tumour proliferation at late stages. Nevertheless, although late-stage Stat3-deficient tumours failed to respond to an anti-CXCL1 monotherapy, the use of CXCL1 blockers combined with standard chemotherapy or as maintenance therapy could be a reasonable alternative to treat these tumours and it deserves further investigation. To substantiate these findings, we inhibited IL-8 signalling in STAT3 knockdown human lung AC cells. Short hairpinmediated IL-8/STAT3 double knockdown resulted in a

Figure 4 | CXCL1 inhibiton reverts oncogenic effects of STAT3 ablation. (a) Mice were treated with the CXCR2 antagonist SB225002 or vehicle control starting 1 week after tumour induction and euthanized after 5 weeks (treatment 1 shown in Supplementary Fig. 5b). Tumour area/lung area was quantified within each group and at least two sections of each lung were stained with haematoxylin and eosin and analysed in a blinded manner. Tumour grading is shown in the right panel ( $n=4-7$ mice per genotype). Data in both panels were analysed by one-way ANOVA with Tukey's multiple comparison test and shown as mean \pm s.e.m. Scale bar, $2 \mathrm{~mm}$. (b) Tumour vascularization was quantified by $\mathrm{CD}_{3} 1^{+}$counts per tumour area (mm²). At least 3 tumours per mouse were analysed with $n=4-7$ mice per genotype and treatment. Data were analysed by one-way ANOVA with Tukey's multiple comparison test and shown as mean \pm s.e.m. Scale bar, $100 \mu \mathrm{m}$. (c) Short hairpin-mediated knockdown of IL-8 (sh/L-8) in either shControl or shSTAT3 A549 NSCLC cells were performed and $2 \times 10^{6}$ cells were injected in both flanks of male nude mice ( $n=5$ per group). Xenograft tumour growth was determined at indicated time points. Data were analysed by Two-way ANOVA with Bonferroni multiple comparison test and shown as mean \pm s.e.m. (d) IHC analysis of CD31+ counts per tumour area $\left(\mathrm{mm}^{2}\right)$ showed reduced vascularization of A549-shSTAT3;sh/L-8 xenograft tumours compared with controls. Data were analysed by one-way ANOVA with Tukey's multiple comparison test and shown as mean \pm s.e.m. Scale bar, $100 \mu \mathrm{m}$. (e) Flow cytometric analysis of Cd11b ${ }^{+} \mathrm{Gr}^{+}$ granulocytes and $\mathrm{Cd11b}{ }^{+} \mathrm{F} 4 / 80^{+}$macrophages displayed reduced myeloid infiltration in A549-shSTAT3;sh/L-8 xenograft tumours compared with controls ( $n \geq 8$ tumours; $5=$ mice per group). At least 6 tumours per mouse were analysed with $n \geq 7$ mice per genotype and treatment. Data were analysed by Kruskal-Wallis test with Dunn's multiple comparison testing and shown as mean \pm s.e.m. For all graphs: ${ }^{\star} P<0.05 ;{ }^{\star \star} P<0.01 ;{ }^{\star \star \star} P<0.001$. 
reduction in tumour growth, tumour vascularization and macrophage infiltration compared with STAT3 single knockdown within the human A549 xenograft model. These results confirm the central role of CXCL1/IL-8 signalling in mutated KRAS STAT3-deficient murine and human xenograft tumours.
Mechanistically, we show that STAT3 signalling can repress NF- $\kappa \mathrm{B}$-driven $C X C L 1 / I L-8$ expression not only in primary mouse pneumocytes but also in human lung AC cells. Furthermore, we show that tumours lacking Stat 3 showed increased nuclear p-p65 concomitant with increased expression of CXCL1 and other typical NF- $\kappa \mathrm{B}$ target genes, such as $\operatorname{Tn} f \alpha, c-M y c$ and Il-6, which a

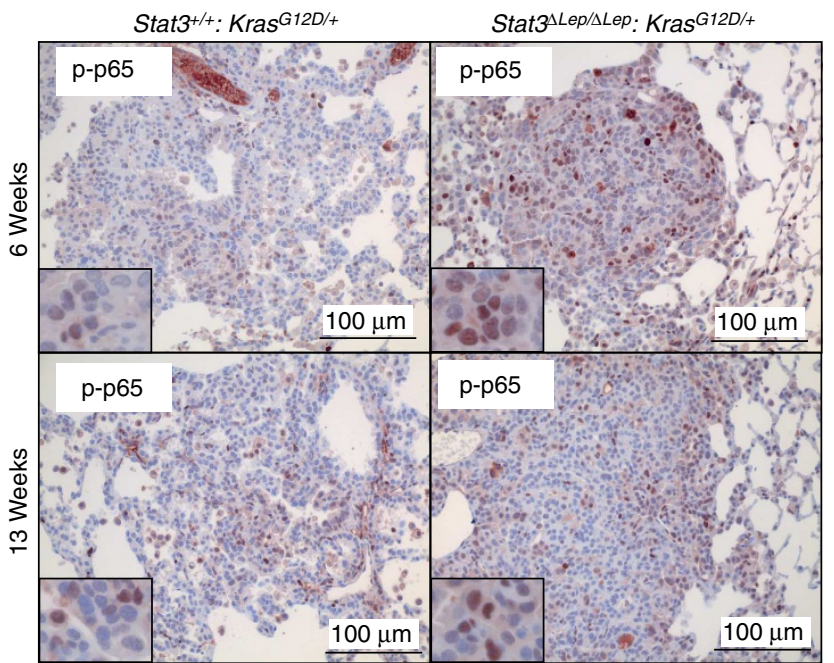

b

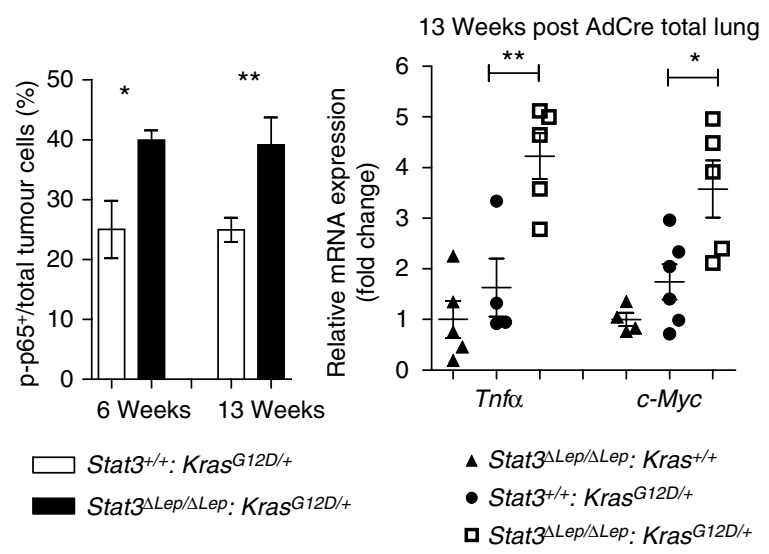

C

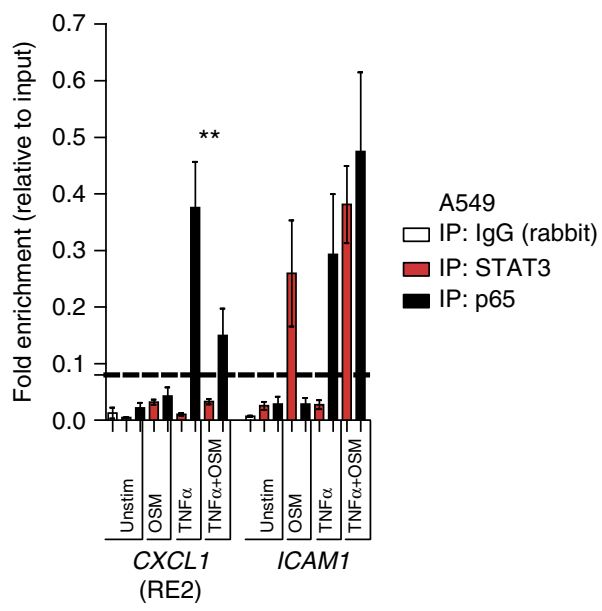

d
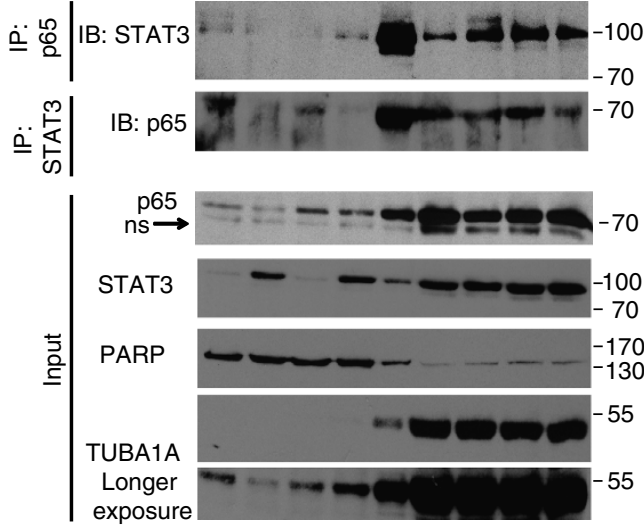

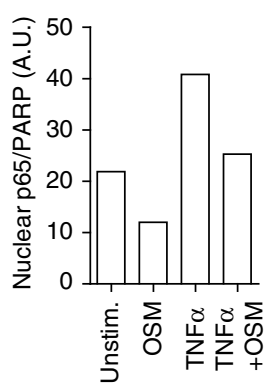

e

p65-FITC

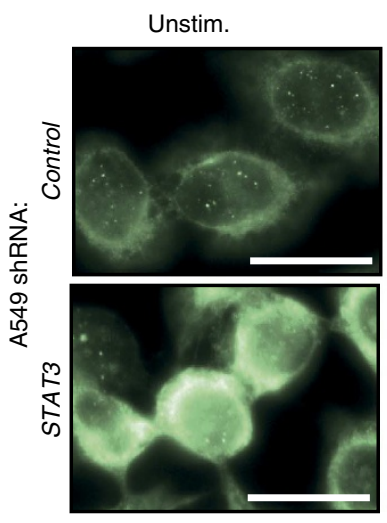

OSM

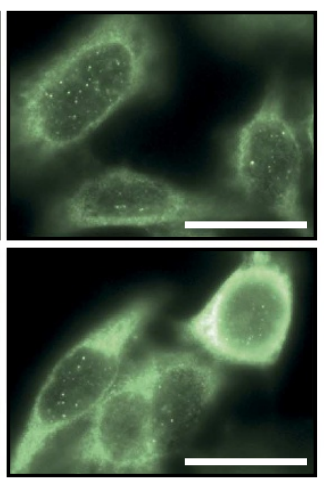

$\mathrm{TNF} \alpha$

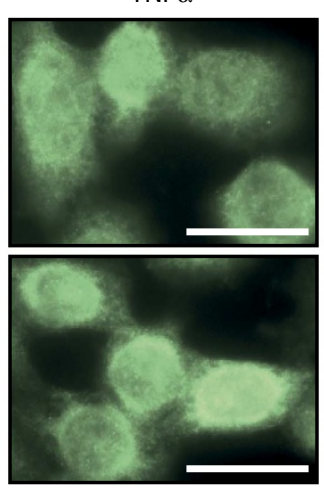

$\mathrm{TNF} \alpha+\mathrm{OSM}$

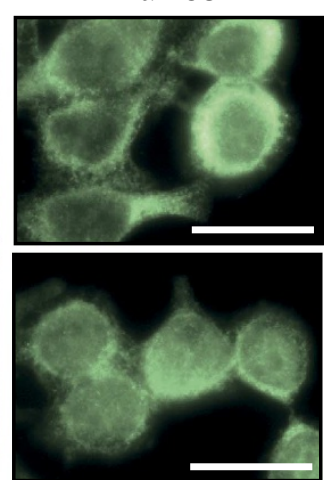

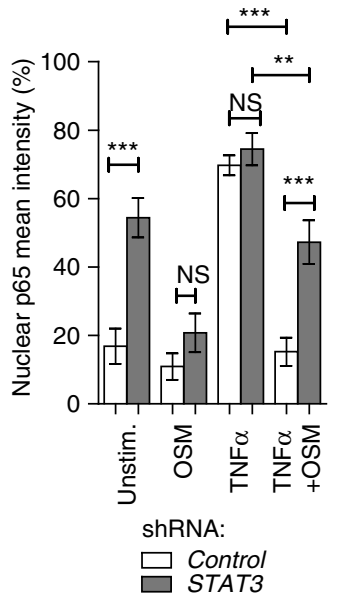


was also shown by others in A549 cells as well as in glioblastoma cells upon STAT3 blockade ${ }^{59,60}$. Although STAT3 as well as other STAT family members has been shown to compete with NF- $\mathrm{KB}$ on various promoter regions $s^{43,61,62}$, we could not detect direct STAT3 binding to the CXCL1 promoter. We found that activation of STAT3 results in binding to and retention of $\mathrm{NF}-\kappa \mathrm{B}$ in the cytoplasm, thereby reducing NF- $\kappa \mathrm{B}$ transcriptional activity. In line with these results, Stat3 deficiency in dendritic cells $^{63}$ and splenocytes as well as knockdown or inhibition of STAT3 within melanoma or prostate cancer cell lines ${ }^{44}$ causes increased phosphorylation of I $\mathrm{I} \mathrm{\alpha} \alpha$, indicating that STAT3 can also impinge on NF- $\mathrm{\kappa B}$ activity further upstream in the inhibitory complex formation of $\mathrm{I} \kappa \mathrm{B} \alpha$ and NF- $\kappa \mathrm{B}$.

Interestingly, mucinous lung AC patients with KRAS mutations not only show reduced STAT3 expression levels but also reduced STAT3 activation levels compared with smoking patients without KRAS mutations and to EGFR mutant cases. Further analysis revealed that more tumours with high granulocyte and low lymphocyte infiltration were found within KRAS mutant patient samples. In addition, low STAT3 expression tends to correlate with increased NF- $\kappa B$ and $I L-8$ expression. Nevertheless, increased patient numbers are needed to substantiate these findings. Furthermore, in lung AC patients with a known smoking history, a patient subgroup associated with KRAS mutations, we found a significant correlation between lower STAT3 expression and poor survival. In line, those tumours with advanced stage (grade III) had significantly less STAT3 expression, indicating that STAT3 downregulation seems to be beneficial for tumour progression. Furthermore, two additional independent data sets confirmed that STAT3 expression was significantly downregulated in high-risk patients with subsequent poor survival outcome in lung AC patients. GSEA further showed that our murine Stat3-deficient Kras ${ }^{G 12 D}$ tumours strongly correlate with a gene expression signature associated with human KRAS mutant lung tumours, and importantly, also with a poor prognosis-gene signature-more than Stat3-proficient Kras ${ }^{G 12 D}$ tumours. These data suggest that our observations in the Stat3deficient $\mathrm{Kras}^{\mathrm{G} 12 \mathrm{D}}$ mouse model are actually reflecting the human situation where tumour grade and poor prognosis correlate with low STAT3 expression in lung AC patients.

Based on in vitro studies and xenograft models, STAT3 is considered to play a tumour-promoting role in EGFR mutant NSCLC $^{13,14,16,18}$, therefore, inhibition of STAT3 is considered as therapeutic intervention in lung $\mathrm{ACs}^{46}$. We have demonstrated that STAT3 has a tumour-suppressor function in murine Kras mutant lung cancer as well as in human KRAS mutant xenografts. Although further clinical investigations will be required, our data suggest that those lung AC patients harbouring KRAS mutations or a known smoking history (as a surrogate marker for KRAS mutations), may not respond to STAT3 inhibitors but may likely suffer severe adverse effects. This highlights the importance of stratifying patients according to their driver mutation for further therapeutic interventions. In addition, our study also proposes a re-evaluation of STAT3 inhibitors ${ }^{46}$ as therapeutic strategies for any inflammatory or fibrotic disease or cancer, as inhibition of STAT3 in vivo might bear the risk of triggering malignant transformation of incipient pre-malignant cells harbouring KRAS mutations within the lung.

\section{Methods}

Tumour induction and inhibitor administration in mice. Mice carrying the Stat $3^{\text {floxed }}$ allele ${ }^{20}$ were crossed with the Kras ${ }^{L S L G 12 D /+}$ knock-in mice ${ }^{19}$ to generate Stat $3^{\text {flox/flox }}:$ Kras $^{G 12 D /+}$ mice maintained on a C57BL6/N background. In all experiments described, littermates were used as controls. All mice were bred and maintained according to an ethical animal licence protocol complying with the current Austrian law. Induction of lung tumours via intranasal AdCre inhalation was performed with $2.5 \times 10^{7}$ plaque-forming unit as described ${ }^{64}$. Stat $3^{\text {4Lep/4Lep }}$ : Kras ${ }^{G 12 D /+}$ and Stat $3^{+/+}:$Kras ${ }^{G 12 D /+}$ mice were treated with SB225002 (Tocris\#2725) as following: 1 week or 6 weeks post Adenoviral Cre infection, mice were injected either with vehicle or SB225002 (dissolved in PBS $+0.25 \%$ Tween-20) with doses of $0.5 \mathrm{mg} \mathrm{kg}^{-1}$ bodyweight intraperitoneally 5 days per week for 5 or 7 weeks, respectively. Treatment was performed according to an animal licence protocol approved by the Bundesministerium für Wissenschaft und Forschung (BMWF-66.009/0252-II/3b/2013).

Xenograft experiments. A total of $2 \times 10^{6}$ cells were mixed 1:2 with Matrigel (Corning\#356231) and injected into left and right flank of Hsd:Athymic NudeFoxn $1^{\text {nu }}$ male mice (3-4 weeks age, Harlan). Tumour volumes were evaluated twice a week by measuring two perpendicular diameters with calipers. Tumour volume was calculated using the following equation: (width*width ${ }^{\star}$ length)/2. Treatment was performed according to an animal licence protocol approved by the Bundesministerium für Wissenschaft und Forschung (BMWF-66.009/0280$\mathrm{II} / 3 \mathrm{~b} / 2012$ ).

\begin{abstract}
Human data. A tissue microarray was produced for mucinous lung ACs. Out of 346 human lung AC cases, 74 were selected that harboured a known smoking history and showed a mucinous differentiation. In comparison to non-mucinous ACs, mucinous subtypes harbour a KRAS mutation in $56 \%$ of cases (versus $25-30 \%$ in non-mucinous AC), thus increasing the probability to identify a significant amount of mutated cases (P.H., unpublished data). Human tissue samples were analysed with prior approval by the institutional ethical committee (24-135 ex $11 / 12$ ). Gene expression data and overall survival information were analysed as described $^{26}$. In brief, the GEO, EGA and TCGA repositories were searched for suitable databases containing raw microarray and survival data as well as clinical characteristics including smoking history for lung AC samples. Two GEO data sets (GSE29013 and GSE31210) fulfilled these criteria. For all samples in these data sets, normalized gene expression was recomputed using MAS5. Then, to analyse the prognostic value of STAT3 or $I L-8$, the lung AC patient samples with or without smoking history were split into two groups according to lower/upper quartile expression of STAT3 or $I L-8$, respectively. The used probe set for STAT3 was 208991_at and for $I L-8$ it was 202859_x_at. The two patient cohorts were analysed by Kaplan-Meier survival plot and the hazard ratio with $95 \%$ confidence intervals and $\log$-rank $P$ value were calculated. The data sets used to determine the prognostic value of STAT3 in the lung AC patients ${ }^{27}$ are taken from GSE30219 and based upon data generated by the TCGA Research Network: http:// cancergenome.nih.gov/.
\end{abstract}

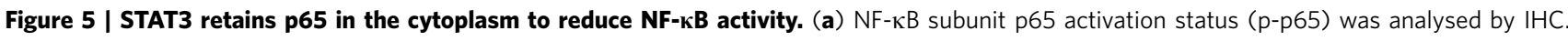

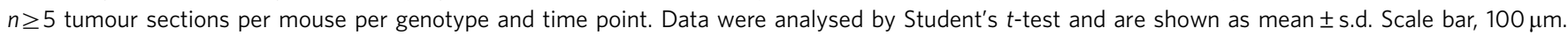

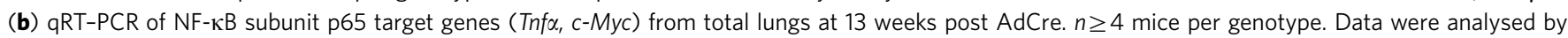
one-way ANOVA with Tukey's multiple comparison test and are displayed as mean \pm s.e.m. (c) Chromatin immunoprecipitation (ChIP) of NF- $\mathrm{B}$

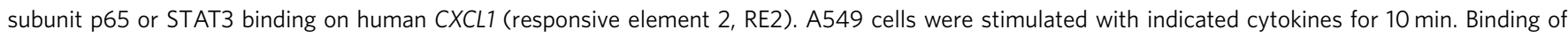
STAT3 and p65 on ICAM1 promoter served as positive control. ACTB element was chosen as negative binding region, indicated by the horizontal line. Values are presented as fold enrichment relative to chromatin input. Data of two independent experiments are shown. Data were analysed by one-way ANOVA with Tukey's multiple comparison test and shown as mean \pm s.e.m. (d) A549 cells were stimulated as in c. Immunoprecipitation (IP) of nuclear and cytoplasmic fractions was performed with antibodies against p65 or STAT3 and subjected to STAT3 and p65 immunoblot analysis, respectively. IP inputs were subjected to STAT3 and p65 (arrow indicates unspecific binding). PARP (nuclear) and TUBA1A (cytoplasmic) were used to determine purity of input. Densitometric quantification of bands was performed (AU, arbitrary units). (e) A549 cells transducted with scrambled shRNA or shRNA against STAT3 were stimulated as in c and stained with antibodies for NF- $\kappa$ B subunit p65 (FITC). $n \geq 6$ regions of interest were quantified. White scale bar, $20 \mu \mathrm{m}$. Data were analysed by one-way ANOVA with Tukey's multiple comparison test and shown as mean \pm s.e.m. For all graphs: ${ }^{\star} P<0.05 ;{ }^{\star \star} P<0.01 ;{ }^{\star \star \star} P<0.001$. 
Histology. For histological analysis of lung tumours, $2.5 \mu \mathrm{m}$ sections from at least two different central planes of the lungs were cut, stained with haematoxylin and eosin and scanned with TissueFaxs software (TissueGnostics $\mathrm{GmbH}$ ). Quantification of tumour area, tumour number and tumour grade was done with HistoQuest software (TissueGnostics $\mathrm{GmbH}$ ) and visually controlled by two independent pathologists (R.H.Z. and H.P.) in a blind manner. Tumour grade was classified as following: hyperplasia includes diffuse hyperplasia of pneumocytes type II and adenomas. The first is a diffuse proliferation, whereas adenomas are circumscribed. Adenomas can be composed of transformed pneumocytes type II but also can contain some Clara cells. Both lesions represent non-malignant proliferations, have a compact cell mass, alveolar septae are still intact and the nuclear/cytoplasmic ratio is still regular. In situ ACs grow along preexisting alveolar septae, have prominent nucleoli and an increased nuclear/cytoplasmic ratio, but still low mitotic counts per high-power field. Sometimes this proliferation appears pseudosolid, because of alveolar collapse or increased cell size obscuring the alveolar lumen. Into this category we also included intrabronchiolar papillary neoplasia as the lesion from which in situ ACs develop. Invasive ACs were classified by harbouring desmoplastic stroma formation (confirmed by Movat pentachrome staining: elastic lamina are stained black-brown; collagen III and V are stained green; collagen I is stained yellow; cells are stained dark red; mucins are stained blue.), invasion into the desmoplastic stroma or lymphatic or blood vessels and high mitotic counts per high-power field.

In situ hybridization and IHC. Digoxigenin labelled RNA fragments for Cxcll were used as probes for in situ hybridization. Human in situ hybridization was performed used the RNAscope 2.0 High Definition assay ${ }^{65}$ according to the manufacturer's instructions (Advanced Cell Diagnostics, Inc.) to detect human IL8 transcripts (Probe-Hs-IL8, 310386). As positive and negative control, we used the RNAscope $H s$-POLR2A (310451) and $\operatorname{dapB}(310043)$ control probe sets. IHC was performed using antibodies against P-STAT3 (Cell Signaling\#9145, 1:100), ser276 p-p65 (Cell Signaling\#3037; 1:50), p65 (Santa Cruz\#372, 1:200), total STAT3 (N-terminal, BD\#610189; 1:200), CD31 (Dianova\#DIA-310, 1:50), SP-C (Santa Cruz\#13979, 1:100) and F4/80 (AbD Serotech\#MCA497G, 1:80), NKp46 (BioLegend\#137602; 1:200), Cd11c (Biolegend 117301, 1:200), CD3 (Neomarkers RM9107, 1:100), FSP1 (Millipore 07-2274, 1:600) using standard protocols. At least five different highpower field sections $(\times 200$ objective $)$ per mouse and per genotype were compared. Quantification for IHC were either done with HistoQuest or for IF staining with TissueQuest (TissueGnostics, GmbH). Image analysis parameters (cell size, cell shape, nuclear size, nuclear shape) were used to discriminate between stroma and tumour cells and were adjusted under the supervision of two board certified pathologist (H.P. and R.H.Z.) to ensure that quantification of P-STAT3 staining in tumour stroma cells versus tumour cells was properly performed.

Microarray analysis. Total RNA from four tumours/genotype at 13 weeks post AdCre were isolated using the RNAeasy Kit (Qiagen\#74104), pooled and hybridized to GeneChip Mouse Gene 1.0 ST array (Affymetrix). GSEA was performed to analyse changes in functional gene sets ${ }^{66}$. Microarray data and description of experimental design are deposited under GEO number GSE52798.

Cytokine measurement. Cytokine Bead Array (eBioscience \#BMS820FF and \#BMS86019FF) was done with the BAL of mice at 13 weeks post AdCre. In short, lungs were instilled and flushed two times with $1 \mathrm{ml}$ sterile phosphate-buffered saline each. The cytokine bead array was performed according to manual instructions, measured with FACS Canto II (BD Biosciences) and analysed with FlowCytomix Pro 2.4 (eBioscience). For lung lysates, a piece of frozen lung was homogenized in phosphate-buffered saline and three freeze-thaw cycles were performed to breakup cellular membrane. Afterwards supernatants were used and ELISA was performed according to the manual instructions (R\&D \#M6000B, \#MEG00, \#MKC00B, \#AF495NA, \#BAF495, \#495MO025; eBioscience \#BMS612). For labelling of infiltrating immune cells, BAL of mice at 6 and 13 weeks post AdCre was used. Single-cell suspensions were labelled overnight at $4{ }^{\circ} \mathrm{C}$ with appropriate antibodies in phosphate-buffered saline. The number of macrophages, granulocytes and MDSCs are defined by the total number of cells in BAL multiplied by the percentage of each cell type identified by flow cytometry (FACS Canto II (BD Biosciences)) and analysed by FlowJo (TreeStar, Inc.).

In vitro experiments and isolation of primary pneumocytes. A549 cells were supplied from ATCC and maintained at standard conditions. STAT3 $\alpha$ overexpressing 3T3 cells and 3T3 control cells were provided by R.M. and described elsewhere ${ }^{10}$. Stimulation experiments were done with human OSM (\#11344022), human IL-6 (\#11340060), human TNF $\alpha$ (\#11343013), murine IL-6 (\#12340063) and murine TNF $\alpha$ (\#352392), all from Immunotools. Murine OSM (\#495MO025) was purchased from R\&D. At least two independent experiments with three individual plates per stimulation were performed. Isolation of primary pneumocytes was performed as described previously ${ }^{22}$. Briefly, lungs were inflated with $3 \mathrm{ml}$ Dispase II (BD\#354235), digested with 0.01\% DNase1 (SIGMA\#D5319) and filtered through 100,70 and $45 \mu \mathrm{m}$ filters. Immune cells were negatively selected with CD16/CD32-coated dishes (BD\#553142). Supernatant containing pneumocytes was maintained with F12 (GIBCO\#VX21765037) media containing penicillin/streptomycin, 2\% FBS, ITS Supplements (SIGMA\#ITSI1884), $0.8 \mathrm{mM}$
$\mathrm{CaCl}_{2}, 15 \mathrm{mM}$ HEPES, $0.25 \%$ BSA. Purity of the batch was confirmed by immunofluorescence staining (Santa Cruz\#13979, 1:100) and nine different highpower fields were quantified by Tissuequest. Forty-eight hours after isolation, cells were infected with calcium precipitated adenoviral particles (University of Iowa, Gene Transfer Vector Core) with a multiplicity of infection (MOI) of 250. Stimulation experiments were performed $120 \mathrm{~h}$ post Adenoviral Cre infection.

Short hairpin-mediated knockdown. Lentivirus production and subsequent transduction of cells was carried out as described earlier ${ }^{67}$. The following shRNA constructs (Sigma Aldrich mission TRC library) were used: shRNA against STAT3 (TRCN0000071456) and control scrambled shRNA (SHC002). The functionality of the shRNAs was validated by immunoblot. Transduced cells were selected for puromycin resistance before further analysis. Within a different approach we used two STAT3-specific shRNAs (\#336, \#946) and a control shRNA (\#713) and cloned them into a retroviral tetracycline-inducible expression vector ${ }^{68}$ containing the optimized miR-E backbone ${ }^{69}$. Retroviral production and subsequent transduction of cells were performed as described earlier ${ }^{68}$. Transduced cells were selected for neomycin resistance and doxycycline was used to induce shRNA expression. Cells were sorted for green fluorescent protein and dsRed prior usage. Short hairpin-mediated knockdown of $I L-8$ was performed by cloning IL-8-specific shRNA (\#1255) into a lentiviral expression vector containing the optimized miR-E backbone ${ }^{69}$. Lentiviral production, transduction and selection with neomycin were performed as described ${ }^{69}$. Cells were sorted for green fluorescent protein in addition to antibiotic selection prior usage.

Chromatin immunoprecipitation. Chromatin isolation was done according to previously described protocol ${ }^{70}$. Briefly, $5 \times 10^{6}$ A549 cells were harvested and fixed in $2 \mathrm{mM}$ Di-succinimidyl Glutarate (Sigma \#80424) for $45 \mathrm{~min}$ at room temperature. After washing, fixation with $1 \%$ formaldehyde was done for $7 \mathrm{~min}$ and stopped with $2.5 \mathrm{M}$ glycine. Chromatin was washed and sonicated in lysis buffer (20\% SDS, 0.5 M EDTA, $1 \mathrm{M}$ Tris $(\mathrm{pH} 8.1), 50 \times$ Proteinase Inhibitor Complete (Roche\#11697498001), 1,000 $\times$ phenylmethylsulphonyl fluoride, $100 \times \mathrm{Na}_{3} \mathrm{Vo}_{4}$ ). Samples were sonicated for 25 cycles at $4{ }^{\circ} \mathrm{C}$; each sonication cycle was for $30 \mathrm{~s}$ followed by a 30 -s pause. $1 \%$ of the chromatin supernatant was kept as input. Immunoprecipitation (IP) was performed with $100 \mu \mathrm{g}$ chromatin incubated with 1:100 diluted antibody (p65\#8242, STAT3\#9134 both Cell signaling, IgG control\#2027 Santa cruz) for $1 \mathrm{~h}$ on ice. Fifty microlitres of pre-cleared beads (Life Technolgies\#10004D) were added per IP and incubated rolling overnight at $4{ }^{\circ} \mathrm{C}$. Next day, chromatin-bead complexes as well as chromatin input were washed with RIPA, high salt, lithium chloride and two times TE buffer before chromatin complexes were eluted in elution buffer (10\%SDS, $0.1 \mathrm{M} \mathrm{NaCO}_{3}, 10 \mathrm{mM}$ dithiothreitol) for $30 \mathrm{~min}$ at room temperature. Reversing the chromatin crosslink was done with $4 \mathrm{M} \mathrm{NaCl}$ at $65^{\circ} \mathrm{C}$ for more than $4 \mathrm{~h}$. The IP complexes were harvested overnight at $55^{\circ} \mathrm{C}$ by adding $0.5 \mathrm{M}$ EDTA, $1 \mathrm{M}$ Tris (pH 6.5) and Proteinase $\mathrm{K}\left(10 \mathrm{mg} \mathrm{ml}^{-1}\right)$. Chromatin-DNA was isolated with Phenol/ Chloroform/Isoamyl Alcohol mixture (Invitrogen\#15593-031) according to protocol. DNA was subjected to quantitative reverse transcription-PCR (qRT-PCR; Kappafirst Peqlab\#07KK600-03) and amount of amplification quantified with standard curves. Primer pairs are listed in Supplementary Table 1.

RNA and real-time quantitative PCR. QRT-PCR was performed on TRIzol (Invitrogen\#15596018) or Qiagen (\#74104) isolated RNA. Complementary DNA transcription was performed either with a Fermentas (\#K1632) or BioRad Kit (\#1708890). qRT-PCR was performed using SYBR Green. Values were normalized to either murine or human $A C T B$ mRNA expression. Primer pairs are listed in Supplementary Table 1.

IP and immunoblotting. Protein lysates and nuclear/cytoplasmic extracts were done according to standard methods. IP was performed overnight with specific antibodies (STAT3 \#9139, p65 \#8242, Cell Signaling) and protein A Sepharose Beads (G\&E\#71709000AE). Immune complexes were dissolved by SDS-polyacrylamide gel electrophoresis and immunoblots were subjected with respective antibodies. Densitometric quantification of immunoblots was performed with the ImageJ software (Rasband, W.S., ImageJ, US National Institutes of Health, Bethesda, Maryland, USA, http://imagej.nih.gov/ij/, 1997-2011.) according to the standard procedures. Cytoplasmic contaminations in the nuclear fraction were corrected using the respective TUBA1A signal before nuclear extract signals were normalized to the PARP-loading control. PARP (\#9532 Cell Signaling), TUBA1A (Santa Cruz\#8035) or HSC-70 (Santa Cruz\#7298) were used as loading controls. Supplementary Fig. $6 \mathrm{~g}$ shows uncropped immunoblots from Fig. 5d.

Statistics. All values are given as means \pm standard error of the mean (s.e.m.) or standard deviation (s.d.) as indicated. Comparisons between two groups were made either by Student's $t$-test or Mann-Whitney $U$-Test; for more than two groups' oneway analysis of variance (ANOVA) with Tukey's multiple comparison test or KruskalWallis test with Dunn's multiple comparison test was performed. Tumour growth was analysed with two-way analysis of variance and Bonferroni multiple comparison testing. For the Kaplan-Meier analysis a log-rank test was performed. $P<0.05$ was accepted as statistically significant. For all graphs: ${ }^{\star} P<0.05 ;{ }^{* *} P<0.01 ;{ }^{* *} P<0.001$. 


\section{References}

1. Jemal, A. et al. Global cancer statistics. CA Cancer J. Clin. 61, 69-90 (2011).

2. Imielinski, M. et al. Mapping the hallmarks of lung adenocarcinoma with massively parallel sequencing. Cell 150, 1107-1120 (2012).

3. Roberts, P. J. \& Stinchcombe, T. E. KRAS mutation: should we test for it, and does it matter? J. Clin. Oncol. 31, 1112-1121 (2013).

4. Gough, D. J. et al. Mitochondrial STAT3 supports Ras-dependent oncogenic transformation. Science 324, 1713-1716 (2009).

5. Lesina, M. et al. Stat3/Socs3 activation by IL-6 transsignaling promotes progression of pancreatic intraepithelial neoplasia and development of pancreatic cancer. Cancer Cell 19, 456-469 (2011).

6. Levy, D. E. \& Darnell, Jr J. E. Stats: transcriptional control and biological impact. Nat. Rev. Mol. Cell Biol. 3, 651-662 (2002).

7. Grivennikov, S. et al. IL-6 and Stat3 are required for survival of intestinal epithelial cells and development of colitis-associated cancer. Cancer Cell 15, 103-113 (2009)

8. Couto, J. P. et al. STAT3 negatively regulates thyroid tumorigenesis. Proc. Natl Acad. Sci. USA 109, E2361-E2370 (2012).

9. de la Iglesia, N. et al. Identification of a PTEN-regulated STAT3 brain tumor suppressor pathway. Genes Dev. 22, 449-462 (2008).

10. Ecker, A. et al. The dark and the bright side of Stat3: proto-oncogene and tumor-suppressor. Front. Biosci. 14, 2944-2958 (2009).

11. Lee, J. et al. Signal transducer and activator of transcription 3 (STAT3) protein suppresses adenoma-to-carcinoma transition in Apcmin/ + mice via regulation of Snail-1 (SNAI) protein stability. J. Biol. Chem. 287, 18182-18189 (2012).

12. Musteanu, M. et al. Stat3 is a negative regulator of intestinal tumor progression in $\mathrm{Apc}(\mathrm{Min})$ mice. Gastroenterology 138, 1003-1011 e1001-1005 (2010).

13. Gao, S. P. et al. Mutations in the EGFR kinase domain mediate STAT3 activation via IL-6 production in human lung adenocarcinomas. J. Clin. Invest. 117, 3846-3856 (2007).

14. Haura, E. B., Zheng, Z., Song, L., Cantor, A. \& Bepler, G. Activated epidermal growth factor receptor-Stat-3 signaling promotes tumor survival in vivo in non-small cell lung cancer. Clin. Cancer Res. 11, 8288-8294 (2005).

15. Song, L., Turkson, J., Karras, J. G., Jove, R. \& Haura, E. B. Activation of Stat3 by receptor tyrosine kinases and cytokines regulates survival in human non-small cell carcinoma cells. Oncogene 22, 4150-4165 (2003)

16. Kluge, A. et al. Cooperative interaction between protein inhibitor of activated signal transducer and activator of transcription-3 with epidermal growth factor receptor blockade in lung cancer. Int. J. Cancer 125, 1728-1734 (2009).

17. Lee, H. J. et al. Drug resistance via feedback activation of Stat 3 in oncogeneaddicted cancer cells. Cancer Cell 26, 207-221 (2014).

18. Weerasinghe, P. et al. Inhibition of Stat3 activation and tumor growth suppression of non-small cell lung cancer by G-quartet oligonucleotides. Int. J. Oncol. 31, 129-136 (2007)

19. Jackson, E. L. et al. Analysis of lung tumor initiation and progression using conditional expression of oncogenic K-ras. Genes Dev. 15, 3243-3248 (2001).

20. Alonzi, T. et al. Essential role of STAT3 in the control of the acute-phase response as revealed by inducible gene inactivation [correction of activation] in the liver. Mol. Cell. Biol. 21, 1621-1632 (2001).

21. Fisher, G. H. et al. Induction and apoptotic regression of lung adenocarcinomas by regulation of a K-Ras transgene in the presence and absence of tumor suppressor genes. Genes Dev. 15, 3249-3262 (2001).

22. Schramek, D. et al. The stress kinase MKK7 couples oncogenic stress to p53 stability and tumor suppression. Nature Genet. 43, 212-219 (2011).

23. Shedden, K. et al. Gene expression-based survival prediction in lung adenocarcinoma: a multi-site, blinded validation study. Nature Med. 14, 822-827 (2008)

24. Sweet-Cordero, A. et al. An oncogenic KRAS2 expression signature identified by cross-species gene-expression analysis. Nature Genet. 37, 48-55 (2005).

25. Sica, G. et al. A grading system of lung adenocarcinomas based on histologic pattern is predictive of disease recurrence in stage I tumors. Am. J. Surg. Pathol. 34, 1155-1162 (2010).

26. Gyorffy, B., Surowiak, P., Budczies, J. \& Lanczky, A. Online survival analysis software to assess the prognostic value of biomarkers using transcriptomic data in non-small-cell lung cancer. PLoS One 8, e82241 (2013).

27. Aguirre-Gamboa, R. et al. SurvExpress: an online biomarker validation tool and database for cancer gene expression data using survival analysis. PLoS One 8, e74250 (2013).

28. Bergers, G. \& Benjamin, L. E. Tumorigenesis and the angiogenic switch. Nat. Rev. Cancer 3, 401-410 (2003).

29. Dong, J. et al. VEGF-null cells require PDGFR alpha signaling-mediated stromal fibroblast recruitment for tumorigenesis. EMBO J. 23, 2800-2810 (2004).

30. Ferrara, N. \& Kerbel, R. S. Angiogenesis as a therapeutic target. Nature 438, 967-974 (2005)

31. Wang, T. et al. Regulation of the innate and adaptive immune responses by Stat-3 signaling in tumor cells. Nat. Med. 10, 48-54 (2004).
32. Murdoch, C., Muthana, M., Coffelt, S. B. \& Lewis, C. E. The role of myeloid cells in the promotion of tumour angiogenesis. Nat. Rev. Cancer. 8, 618-631 (2008).

33. Youn, J. I. \& Gabrilovich, D. I. The biology of myeloid-derived suppressor cells the blessing and the curse of morphological and functional heterogeneity. Eur J. Immunol. 40, 2969-2975 (2010).

34. Zlotnik, A., Yoshie, O. \& Nomiyama, H. The chemokine and chemokine receptor superfamilies and their molecular evolution. Genome Biol. 7, 243 (2006).

35. Petreaca, M. L., Yao, M., Liu, Y., Defea, K. \& Martins-Green, M. Transactivation of vascular endothelial growth factor receptor-2 by interleukin8 (IL-8/CXCL8) is required for IL-8/CXCL8-induced endothelial permeability. Mol. Biol. Cell 18, 5014-5023 (2007).

36. Waugh, D. J. \& Wilson, C. The interleukin-8 pathway in cancer. Clin. Cancer Res. 14, 6735-6741 (2008)

37. Sunaga, N. et al. Oncogenic KRAS-induced interleukin-8 overexpression promotes cell growth and migration and contributes to aggressive phenotypes of non-small cell lung cancer. Int. J. Cancer 130, 1733-1744 (2012).

38. Ijichi, H. et al. Inhibiting Cxcr2 disrupts tumor-stromal interactions and improves survival in a mouse model of pancreatic ductal adenocarcinoma. J. Clin. Invest. 121, 4106-4117 (2011).

39. Richmond, A. Nf-kappa B, chemokine gene transcription and tumour growth. Nat. Rev. Immunol. 2, 664-674 (2002).

40. Sparmann, A. \& Bar-Sagi, D. Ras-induced interleukin-8 expression plays a critical role in tumor growth and angiogenesis. Cancer Cell 6, 447-458 (2004).

41. Basseres, D. S. \& Baldwin, A. S. Nuclear factor-kappaB and inhibitor of kappaB kinase pathways in oncogenic initiation and progression. Oncogene 25, 6817-6830 (2006).

42. de la Iglesia, N. et al. Deregulation of a STAT3-interleukin 8 signaling pathway promotes human glioblastoma cell proliferation and invasiveness. J. Neurosci. 28, 5870-5878 (2008).

43. Yu, Z., Zhang, W. \& Kone, B. C. Signal transducers and activators of transcription 3 (STAT3) inhibits transcription of the inducible nitric oxide synthase gene by interacting with nuclear factor kappaB. Biochem. J. 367, 97-105 (2002).

44. Lee, H. et al. Persistently activated Stat3 maintains constitutive NF-kappaB activity in tumors. Cancer Cell 15, 283-293 (2009).

45. Yang, J. et al. Novel roles of unphosphorylated STAT3 in oncogenesis and transcriptional regulation. Cancer Res. 65, 939-947 (2005).

46. Debnath, B., Xu, S. \& Neamati, N. Small molecule inhibitors of signal transducer and activator of transcription 3 (Stat3) protein. J. Med. Chem. 55, 6645-6668 (2012).

47. Gordziel, C., Bratsch, J., Moriggl, R., Knosel, T. \& Friedrich, K. Both STAT1 and STAT3 are favourable prognostic determinants in colorectal carcinoma. Br. J. Cancer 109, 138-146 (2013).

48. Sonnenblick, A. et al. Tissue microarray-based study of patients with lymph node-positive breast cancer shows tyrosine phosphorylation of signal transducer and activator of transcription 3 (tyrosine705-STAT3) is a marker of good prognosis. Clin. Transl. Oncol. 14, 232-236 (2012).

49. Ihara, S. et al. Inhibitory roles of signal transducer and activator of transcription 3 in antitumor immunity during carcinogen-induced lung tumorigenesis. Cancer Res. 72, 2990-2999 (2012).

50. You, S. et al. Disruption of STAT3 by niclosamide reverses radioresistance of human lung cancer. Mol. Cancer Ther. 13, 606-616 (2014).

51. Zhang, X., Zhang, J., Wang, L., Wei, H. \& Tian, Z. Therapeutic effects of STAT3 decoy oligodeoxynucleotide on human lung cancer in xenograft mice. $B M C$ Cancer 7, 149 (2007).

52. Mostertz, W. et al. Age- and sex-specific genomic profiles in non-small cell lung cancer. JAMA 303, 535-543 (2010).

53. Naugler, W. E. et al. Gender disparity in liver cancer due to sex differences in MyD88-dependent IL-6 production. Science 317, 121-124 (2007).

54. Wang, H. C. et al. Activation of the signal transducer and activator of transcription 3 pathway up-regulates estrogen receptor-beta expression in lung adenocarcinoma cells. Mol. Endocrinol. 25, 1145-1158 (2011).

55. Saintigny, P. et al. CXCR2 expression in tumor cells is a poor prognostic factor and promotes invasion and metastasis in lung adenocarcinoma. Cancer Res. 73, 571-582 (2013).

56. Galffy, G. et al. Interleukin 8: an autocrine growth factor for malignant mesothelioma. Cancer Res. 59, 367-371 (1999).

57. Luppi, F., Longo, A. M., de Boer, W. I., Rabe, K. F. \& Hiemstra, P. S. Interleukin-8 stimulates cell proliferation in non-small cell lung cancer through epidermal growth factor receptor transactivation. Lung Cancer 56, 25-33 (2007)

58. Zhu, Y. M., Webster, S. J., Flower, D. \& Woll, P. J. Interleukin-8/CXCL8 is a growth factor for human lung cancer cells. Br. J. Cancer 91, 1970-1976 (2004)

59. Kulesza, D. W., Carre, T., Chouaib, S. \& Kaminska, B. Silencing of the transcription factor STAT3 sensitizes lung cancer cells to DNA damaging drugs, but not to TNFalpha- and NK cytotoxicity. Exp. Cell Res. 319, 506-516 (2013). 
60. McFarland, B. C., Gray, G. K., Nozell, S. E., Hong, S. W. \& Benveniste, E. N. Activation of the NF-kappaB pathway by the STAT3 inhibitor JSI-124 in human glioblastoma cells. Mol. Cancer Res. 11, 494-505 (2013).

61. Bennett, B. L., Cruz, R., Lacson, R. G. \& Manning, A. M. Interleukin-4 suppression of tumor necrosis factor alpha-stimulated E-selectin gene transcription is mediated by STAT6 antagonism of NF-kappaB. J. Biol. Chem. 272, 10212-10219 (1997).

62. Zhang, Z. \& Fuller, G. M. Interleukin 1beta inhibits interleukin 6-mediated rat gamma fibrinogen gene expression. Blood 96, 3466-3472 (2000).

63. Welte, T. et al. STAT3 deletion during hematopoiesis causes Crohn's diseaselike pathogenesis and lethality: a critical role of STAT3 in innate immunity. Proc. Natl Acad. Sci. USA 100, 1879-1884 (2003).

64. DuPage, M., Dooley, A. L. \& Jacks, T. Conditional mouse lung cancer models using adenoviral or lentiviral delivery of Cre recombinase. Nat. Protoc. 4, 1064-1072 (2009).

65. Wang, F. et al. RNAscope: a novel in situ RNA analysis platform for formalin-fixed, paraffin-embedded tissues. J. Mol. Diagn. 14, 22-29 (2012).

66. Subramanian, A. et al. Gene set enrichment analysis: a knowledge-based approach for interpreting genome-wide expression profiles. Proc. Natl Acad. Sci. USA 102, 15545-15550 (2005).

67. Kasper, M., Regl, G., Eichberger, T., Frischauf, A. M. \& Aberger, F. Efficient manipulation of Hedgehog/GLI signaling using retroviral expression systems. Methods Mol. Biol. 397, 67-78 (2007).

68. Zuber, J. et al. Toolkit for evaluating genes required for proliferation and survival using tetracycline-regulated RNAi. Nature Biotechnol. 29, 79-83 (2011).

69. Fellmann, C. et al. An optimized microRNA backbone for effective single-copy RNAi. Cell Rep. 5, 1704-1713 (2013).

70. Porro, A. \& Perini, G. In Vivo Dual Cross-Linking Chromatin Immunoprecipitation: Detecting Chromatin Proteins not Directly Bound to DNA (PROT 29) (The Epigenome-Network of Excellence: Epigenetics protocols database., 2007).

\section{Acknowledgements}

We thank all members of our institute for discussions; M. Jeitler and M. Bilban from the Core Facility Genomics of the Medical University of Vienna for microarray performance. M. Musteanu, B. Pranjic, M. Prchal-Murphy and M. Schlederer for scientific support.
The project was supported by GENAU grant 'Austromouse' and the Austrian Science Fund (FWF): P 25599.

\section{Author contributions}

B.G. designed and performed most experiments. J.S., L.B. performed in situ hybridization. K.M.M., E.B., K.Z., N.H., P.S., E.Bo., H.N. and J.S. provided major technical support. W.G., F.A., T.H. and J.Z. provided and helped with shRNA knockdown. R.H.Z. and H.P. provided major histological support. H.P. provided human data sets. H.E. and T.M. helped in microarray and gene set enrichment analysis. D.S., K.M.M., G.E., B.Gy., H.E., R.E. and H.P. assisted in data interpretation. All other co-authors gave scientific support. E.C. coordinated the project. Manuscript was written by B.G., K.M.M, H.P., H.P.M., D.S. and E.C.

\section{Additional information}

Accession codes: Microarray data and description of experimental design are deposited under GEO number GSE52798. GSE29013 and GSE31210 were used for acquiring data from Fig. 1g and Supplementary Fig. 2c. In Fig. 1h,i and Supplementary Fig. 2d,e, GSE30219 and data generated by the TCGA Research Network: http://cancergenome. nih.gov/ were used.

Supplementary Information accompanies this paper at http://www.nature.com/ naturecommunications

Competing financial interests: The authors declare no competing financial interests.

Reprints and permission information is available online at http://npg.nature.com/ reprintsandpermissions/

How to cite this article: Grabner, B. et al. Disruption of STAT3 signalling promotes KRAS-induced lung tumorigenesis. Nat. Commun. 6:6285 doi: 10.1038/ncomms7285 (2015).

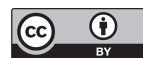

This work is licensed under a Creative Commons Attribution 4.0 International License. The images or other third party material in this article are included in the article's Creative Commons license, unless indicated otherwise in the credit line; if the material is not included under the Creative Commons license, users will need to obtain permission from the license holder to reproduce the material. To view a copy of this license, visit http://creativecommons.org/licenses/by/4.0/ 